\title{
The Relation Between Nutritional Habits and Social Factors on School Performance of Students of Elementary Schools in Fayoum
}

\author{
Hanan A. Hussien, Nabih A. Ibrahim, Amal Hassanin \\ Food Technology Research Institute, Agricultural Research Centre, Cairo, Egypt
}

\section{Email address:}

hananhussienh@yahoo.com (H. A. Hussien)

\section{To cite this article:}

Hanan A. Hussien, Nabih A. Ibrahim, Amal Hassanin. The Relation Between Nutritional Habits and Social Factors on School Performance of Students of Elementary Schools in Fayoum. Journal of Food and Nutrition Sciences. Vol. 3, No. 2, 2015, pp. 71-83.

doi: 10.11648/j.jfns.20150302.17

\begin{abstract}
Background: Malnutrition among children in developing countries continues to impair health and development. School feeding is supposed to eliminate hunger of school age children improving their health, education and future productivity as adults. Objective: to assess the effect of breakfast and school feeding program (SFP) on the academic performance of primary school children in Fayoum governorate. Methods: The intervention group consisted of 576 pupils in the 5th grade chosen randomly from schools have taken school meal for five years. The control group was 233 age and sex-matched pupils chosen randomly from schools have never taken school meal. The snack consists of a pie fortified with minerals and vitamins. Assessment of nutritional status, socio-demographic variables and dietary behaviour was performed. Grades of pupils were taken. Results: Children, who have got school meal for five years, $81.8 \%$ and $88.7 \%$ of them passed in school and math respectively, for girls the effect was higher. Other nutritional factors have less positive effect. Social factors have less effect on both school achievement and math. Multiple regression analysis presents school meal as the main predictor of school achievement and math grades, while sex and family income are the only socioeconomic variables predictors of school achievement and math grades. Conclusion: School snack has the opportunity to alleviate short-term hunger during the school day. Providing students with important micronutrients especially iron, regularly through daily intake of school snacks, results in improvement in school grades. School meal should be one of multiple interventions designed to improve the environment in which poor children live and learn.
\end{abstract}

Keywords: School Meal, Breakfast, Micronutrients, Math Grades, School Achievements

\section{Introduction}

Nutrition, or the lack of it, has recently been recognized as an important additional factor because studies have identified under-nutrition with poor school achievement (Powell \& Grantham-McGregor, 1980; Grantham-McGregor et al., 1998; Ahmed, 2004).Iron deficiency in schoolchildren also reduces some aspects of their ability to learn and providing iron improves these capacities. Hook worm infection, one cause of iron deficiency anemia and malnutrition, is also linked to diminished learning capacity (Acham et al., 2012).

Children who are already at risk due to nutritionals inadequacy come to school tired, hungry, or hurting, unable to cope and benefit from their lessons (Ani\& GranthamMcGregor, 1999). Absenteeism, delayed enrolment, lowered cognitive capacity, and reduced academic achievement are all associated with either an over or under supply of important nutrients (Grantham- McGregor, 2002). We still do not fully understand the actual mechanisms linking nutrition to learning, or even IQ. But, it can be said that malnutrition brings with it harmful consequences for body and mind alike (Gorman, 1995; Grantham-McGregor, 1995; Levitsky \& Strupp, 1995; Wachs, 1995; Grantham-McGregor et al., 2000).

One of the most common and wide spread nutritional insults to children is iron deficiency. Severe anaemia is associated with increased childhood mortality, and mild to moderate anaemia is associated with lowered work capacity and productivity, immune function, altered behaviour, and cognitive development (Bhaskaram, 2001; Neumann et al., 
2002).

Ekaju's study (2011)citing the World Bank (2011) notes that programs that address hunger, malnutrition, and disease among school children significantly improve their academic performance, a reason why school-based feeding and health programs can be valuable.

The aim of this study was to assess and demonstrate the effect of breakfast and midday meal consumption on academic achievement of primary schoolchildren (grade 5) in Fayoum governorate, Upper Egypt.

\section{Subjects and Methods}

\subsection{Study Design}

The static group comparison design was employed as there was no baseline data to apply Pretest- posttest control design; the ideal to achieve our objective (Trochim, 2006) Egypt's Ministry of education used a "poverty map" to determine which schools have the priority to receive school meals in each governorate. The evaluation covered a period of eighteen months starting from October 2009 till May 2011. This cross-sectional study was conducted in Fayoum representing rural, urban areas.

The intervention group consisted of 569 pupils in the 5 th grade chosen randomly from schools that have taken school meal for five years. The control group was 240 age and sexmatched pupils chosen randomly from schools that have never taken school meal.

Inclusion criteria: apparently healthy prepubescent boys and girls in the 5 th grade.

Exclusion criteria: Students proved to have any mental disorder, chronic disease, visual or auditory impairment, were excluded.

The study was approved by the Ethical Committees of the National Research Centre and Agricultural Research Centre. Permission to conduct the research in the selected schools was also obtained from the Egyptian Ministry of Education. Data were collected between March 2010 and May 2010.

\section{Methods}

A well-structured questionnaire: was applied to each student through an interview to collect socio-demographic and nutritional data. The information collected included age, gender, number of family members, birth order of the child under study, parent's education level, parent's occupation, and household income.

\subsection{Dietary Pattern Assessment}

To evaluate dietary habits and behaviour of children (skipping breakfast, number of meals consumed per day, eating of the served school meal, source of other meals consumed through school day).

The students' grades in math were collected for first terms from official school records.

\subsection{Statistical Analysis}

Data were analyzed using Statistical Package for Social Sciences (SPSS) version 16. Quantitative Data were presented as means $\pm \mathrm{SD}$. The independent sample-t test was used to analyze mean difference. Qualitative data were summarized as percentages and comparison between groups was done using Chi square-test. For all tests, probability values $(\mathrm{P})$ of less than 0.05 were regarded as statistically significant. ANOVA with Post-hoc test was used to study the association of cognitive function and the intake of school snack and breakfast eating pattern. Multiple linear regression analysis was done to assess the contribution of each independent variable (SFP, socio-economic status, breakfast eating habit, and nutritional status) in explaining the variation in the dependent variables.

\section{Results}

School Feeding Program (SFP) was established by the Ministry of Agriculture and Land Reclamation (MOLAR) in Egypt, to attract children in disadvantaged rural and urban communities to attend primary schools. Nutritious fortified snacks in the form of sweet pies were provided to those children in the mid-morning, to supply them with nutrients essential to their development and concentration ability. A field evaluation of the effectiveness of SFP was conducted by the collaboration between Agricultural Research Center and National Research Center in Egypt.

\subsection{Composition of the School Snack}

The snack consists of a pie fortified with minerals and vitamins. Elements in the fortified flour are: Vitamin A, vitamin $C$, thiamin, riboflavin, vitamin $B_{6}$ and $B_{12}$, niacin, folate, calcium, iron, zinc and phosphorus. Although the school meal provides only $16.9 \%$ of RDA for energy, $4.3 \%$ of RDA for vitamin C; $5.5 \%$ of RDA for calcium; yet it provides $35.8 \%$ of RDA for iron and $41.7 \%$ of RDA for folate, and about $26.81 \%$ of $\mathrm{RDA}$ for protein. The pie contains either minced dry date or a jam to be more nutritious and acceptable.

Table 1. Analysis of the School Meal.

\begin{tabular}{llll}
\hline & RDA & Pie & \%RDA \\
\hline Energy & 2000 & 338.067 & 16.90 \\
Protein & 28 & 7.506 & 26.81 \\
Vit A & 3300 & 332.629 & 10.08 \\
Vit C & 45 & 1.923 & 4.27 \\
Thiamin & 1 & 0.094 & 9.40 \\
Riboflavin & 1.2 & 0.141 & 11.75 \\
Vit B6 & 1.2 & 0.053 & 4.42 \\
Folate & 100 & 41.762 & 41.76 \\
Niacin & 13 & 1.03 & 7.92 \\
Vit B12 & 1.4 & 0.122 & 8.71 \\
Calcium & 800 & 44.477 & 5.56 \\
Zinc & 10 & 0.692 & 6.92 \\
Iron & 10 & 3.588 & 35.88 \\
Phosphorus & 800 & 109.138 & 13.64 \\
\hline
\end{tabular}




\subsection{Characteristics of the Participants}

Most of the participants were aged 11 to 12 years, of which most (70.6\%) lived in relatively large households (with more than 5 people). More than half $(65.4 \%)$ were born to mothers who had either no formal education or had incomplete primary education, $(50.3 \%)$ were born to fathers who had either no formal education or had incomplete primary education.

\subsection{Effect of Nutritional Factors on Academic Performance}

It can be noticed that school meal has a direct positive and significant effect on passing school. $81.8 \%$ of children receiving the school meal passed in school exams compared with only $51.9 \%$ of children not having a meal $(\mathrm{OR}=2.83)$.
Having the school meal in the morning had positive significant effect on passing school $(\mathrm{OR}=2.78$ and $\mathrm{OR}=4.92)$ compared with the afternoon and no specific time respectively. Also, the regularity of meals and the mid-day snack have positive, but less, significant effect on passing school exams $(\mathrm{OR}=0.62$ and 1.71) respectively. A combination of breakfast and school meal has a highly positive effect $(\mathrm{OR}=4.92)$. Other nutritional factors have no significant effect on passing school exams. Our results agree with previous work by Adrogué and Orlicki, 2013, who reported that eating breakfast, in contrast to fasting, may improve performance on the morning eaten. The long-term effects of eating breakfast on the performance of school children who do not have physical signs of severe undernourishment are less certain.

Table 2. School Achievement for Boys, Girls and the Combined Group vs. some Nutritional Factors.

\begin{tabular}{|c|c|c|c|c|c|c|c|c|c|c|c|c|}
\hline & \multicolumn{4}{|l|}{ Boys } & \multicolumn{4}{|c|}{ Girls } & \multicolumn{4}{|c|}{ Combined } \\
\hline & $\mathbf{n}$ & $\begin{array}{l}\text { Failed } \\
<50\end{array}$ & $\begin{array}{l}\text { Passed } \\
>\mathbf{5 0}\end{array}$ & $\begin{array}{l}\mathrm{X}^{2} \\
\text { (sig) }\end{array}$ & $\mathbf{n}$ & $\begin{array}{l}\text { Failed } \\
<50\end{array}$ & $\begin{array}{l}\text { Passed } \\
>\mathbf{5 0}\end{array}$ & $\begin{array}{l}\mathrm{X}^{2} \\
\text { (sig) }\end{array}$ & $\mathbf{n}$ & $\begin{array}{l}\text { Failed } \\
<\mathbf{5 0}\end{array}$ & $\begin{array}{l}\text { Passed } \\
>\mathbf{5 0}\end{array}$ & $\begin{array}{l}\mathrm{X}^{2} \\
\text { (sig) }\end{array}$ \\
\hline \multicolumn{13}{|c|}{ Receiving School Meal } \\
\hline No Meal & 120 & $\begin{array}{l}66 \\
(55.0 \%)\end{array}$ & $\begin{array}{l}54 \\
(45.0 \%)\end{array}$ & 43.39 & 120 & $\begin{array}{l}51 \\
(42.5 \%)\end{array}$ & $\begin{array}{l}69 \\
(57.5 \%)\end{array}$ & 42.33 & 240 & $\begin{array}{l}112 \\
(48.1 \%)\end{array}$ & $\begin{array}{l}121 \\
(51.9 \%)\end{array}$ & 43.55 \\
\hline \multirow[t]{2}{*}{ Meal } & 293 & $\begin{array}{l}64 \\
(21.8 \%)\end{array}$ & $\begin{array}{l}229 \\
(78.2 \%)\end{array}$ & $(0.000)$ & 276 & $\begin{array}{l}36 \\
(13.0 \%)\end{array}$ & $\begin{array}{l}240 \\
(87.0 \%)\end{array}$ & $(0.000)$ & 569 & $\begin{array}{l}154 \\
(18.2 \%)\end{array}$ & $\begin{array}{l}471 \\
(81.8 \%)\end{array}$ & $(0.000)$ \\
\hline & \multicolumn{3}{|c|}{$\mathrm{OR}=4.37(2.78-6.89)$} & & \multicolumn{4}{|c|}{$\mathrm{OR}=4.93(2.98-8.15)$} & \multicolumn{3}{|c|}{$\mathrm{OR}=2.83(2.04-3.93)$} & \\
\hline \multicolumn{13}{|c|}{ Regularity of Meals } \\
\hline No & 123 & $\begin{array}{l}29 \\
(23.6 \%)\end{array}$ & $\begin{array}{l}94 \\
(76.4 \%)\end{array}$ & 5.07 & 132 & $\begin{array}{l}24 \\
(18.2 \%)\end{array}$ & $\begin{array}{l}108 \\
(81.8 \%)\end{array}$ & 1.657 & 255 & $\begin{array}{l}53 \\
(20.8 \%)\end{array}$ & $\begin{array}{l}202 \\
(79.2 \%)\end{array}$ & 6.92 \\
\hline \multirow[t]{2}{*}{ Yes } & 290 & $\begin{array}{l}101 \\
(34.8 \%)\end{array}$ & $\begin{array}{l}189 \\
(65.2 \%)\end{array}$ & 0.02 & 264 & $\begin{array}{l}63 \\
(23.9 \%)\end{array}$ & $\begin{array}{l}201 \\
(76.1 \%)\end{array}$ & 0.123 & 554 & $\begin{array}{l}164 \\
(29.6 \%)\end{array}$ & $\begin{array}{l}390 \\
(70.4 \%)\end{array}$ & $0.008)$ \\
\hline & \multicolumn{3}{|c|}{$\mathrm{OR}=0.58(0.36-0.93)$} & & \multicolumn{3}{|c|}{$\mathrm{OR}=0.71(0.42-1.20)$} & & \multicolumn{3}{|c|}{$\mathrm{OR}=0.62(0.44-0.89)$} & \\
\hline \multicolumn{13}{|l|}{ Mid-Day Snack } \\
\hline No & 60 & $\begin{array}{l}21 \\
(35.0 \%)\end{array}$ & $\begin{array}{l}39 \\
(65.0 \%)\end{array}$ & 0.40 & 35 & $\begin{array}{l}14 \\
(40.0 \%)\end{array}$ & $\begin{array}{l}21 \\
(60.0 \%)\end{array}$ & 7.281 & 95 & $\begin{array}{l}35 \\
(36.8 \%)\end{array}$ & $\begin{array}{l}60 \\
(63.2 \%)\end{array}$ & 5.50 \\
\hline \multirow[t]{2}{*}{ Yes } & 353 & $\begin{array}{l}109 \\
(30.9 \%)\end{array}$ & $\begin{array}{l}244 \\
(69.1 \%)\end{array}$ & $(0.53)$ & 361 & $\begin{array}{l}73 \\
(20.2 \%)\end{array}$ & $\begin{array}{l}288 \\
(79.8 \%)\end{array}$ & $(0.009)$ & 714 & $\begin{array}{l}182 \\
(25.5 \%)\end{array}$ & $\begin{array}{l}532 \\
(74.5 \%)\end{array}$ & $(0.02)$ \\
\hline & \multicolumn{3}{|c|}{$\mathrm{OR}=1.21(0.68-2.15)$} & & \multicolumn{3}{|c|}{$\mathrm{OR}=2.63(1.26-5.42)$} & & \multicolumn{3}{|c|}{$\mathrm{OR}=1.71(1.09-2.67)$} & \\
\hline \multicolumn{13}{|c|}{ Time of Distribution \& Eating School Meal } \\
\hline Afternoon & 32 & $\begin{array}{l}10 \\
(31.2 \%)\end{array}$ & $\begin{array}{l}22 \\
(68.8 \%)\end{array}$ & 4.76 & 139 & $\begin{array}{l}17 \\
(12.2 \%)\end{array}$ & $\begin{array}{l}122 \\
(87.8 \%)\end{array}$ & 0.05 & 56 & $\begin{array}{l}13 \\
(23.2 \%)\end{array}$ & $\begin{array}{l}43 \\
(76.8 \%)\end{array}$ & 3.81 \\
\hline \multirow[t]{2}{*}{ Morning } & 63 & $\begin{array}{l}8 \\
(12.7 \%)\end{array}$ & $\begin{array}{l}55 \\
(87.3 \%)\end{array}$ & $(0.03)$ & 63 & $\begin{array}{l}7 \\
(11.1 \%)\end{array}$ & $\begin{array}{l}56 \\
(88.9 \%)\end{array}$ & $(0.82)$ & 126 & $\begin{array}{l}15 \\
(11.9 \%)\end{array}$ & $\begin{array}{l}111 \\
(88.1 \%)\end{array}$ & $(0.05)$ \\
\hline & \multicolumn{4}{|c|}{$\mathrm{OR}=3.13(1.09-8.96)$} & \multicolumn{4}{|c|}{$\mathrm{OR}=1.11(0.44-2.84)$} & \multicolumn{3}{|c|}{$\mathrm{OR}=2.24(0.98-5.09)$} & \\
\hline \multicolumn{13}{|c|}{ Time of Distribution \& Eating School Meal } \\
\hline $\begin{array}{l}\text { No Specific } \\
\text { Time }\end{array}$ & 41 & $\begin{array}{l}15 \\
(36.6 \%)\end{array}$ & $\begin{array}{l}26 \\
(63.4 \%)\end{array}$ & 8.23 & 47 & $\begin{array}{l}9 \\
(19.1 \%)\end{array}$ & $\begin{array}{l}38 \\
(80.9 \%)\end{array}$ & 1.40 & 88 & $\begin{array}{l}24 \\
(27.3 \%)\end{array}$ & $\begin{array}{l}64 \\
(72.7 \%)\end{array}$ & 8.21 \\
\hline \multirow[t]{2}{*}{ Morning } & 63 & $\begin{array}{l}8 \\
(12.7 \%)\end{array}$ & $\begin{array}{l}55 \\
(87.3 \%)\end{array}$ & $(0.004)$ & 63 & $\begin{array}{l}7 \\
(11.1 \%)\end{array}$ & $\begin{array}{l}56 \\
(88.9 \%)\end{array}$ & $(0.24)$ & 126 & $\begin{array}{l}15 \\
(11.9 \%)\end{array}$ & $\begin{array}{l}111 \\
(88.1 \%)\end{array}$ & $(0.004)$ \\
\hline & \multicolumn{4}{|c|}{$\mathrm{OR}=3.97(1.49-10.53)$} & \multicolumn{4}{|c|}{$\mathrm{OR}=1.89(0.65-5.53)$} & $\mathrm{OR}=$ & $78(1.36-5$ & & \\
\hline School Meal \& & 3reakf: & & & & & & & & & & & \\
\hline $\begin{array}{l}\text { No School } \\
\text { Meal\& No } \\
\text { Breakfast }\end{array}$ & 40 & $\begin{array}{l}31 \\
(77.5 \%)\end{array}$ & $\begin{array}{l}9 \\
(22.5 \%)\end{array}$ & 76.54 & 48 & $\begin{array}{l}23 \\
(47.5 \%)\end{array}$ & $\begin{array}{l}25 \\
(52.1 \%)\end{array}$ & 27.01 & 88 & $\begin{array}{l}46 \\
(52.27 \%)\end{array}$ & $\begin{array}{l}42 \\
(47.73 \%)\end{array}$ & 45.88 \\
\hline $\begin{array}{l}\text { School } \\
\text { Meal\& } \\
\text { Breakfast }\end{array}$ & 224 & $\begin{array}{l}31 \\
(13.8 \%)\end{array}$ & $\begin{array}{l}193 \\
(86.2 \%)\end{array}$ & $(0.000)$ & 188 & $\begin{array}{l}26 \\
(13.8 \%)\end{array}$ & $\begin{array}{l}162 \\
(86.2 \%)\end{array}$ & $(0.000)$ & 412 & $\begin{array}{l}75 \\
(18.2 \%)\end{array}$ & $\begin{array}{l}337 \\
(81.8 \%)\end{array}$ & $(0.000)$ \\
\hline & $\mathrm{OR}=$ & $.44(9.32-2$ & 35) & & $\mathrm{OR}=$ & $73(2.84-1)$ & & & $\mathrm{OR}=$ & $92(3.02-8$. & & \\
\hline
\end{tabular}

Results from table 2 shows that, when comparing the effect of nutritional factors on boys and girls in the sample, there is a difference in factors affecting the school passing performance. In girls, having a meal has a significant positive effect which is higher $(\mathrm{OR}=4.93)$ compared with boys $(\mathrm{OR}=$ 4.37). Also, the mid-day snack has a significant positive effect $(\mathrm{OR}=2.63)$ and the combination of breakfast and school meal $(\mathrm{OR}=5.73)$. As for boys, having a meal has a significant 
positive effect which is lower $(\mathrm{OR}=4.37)$ compared with girls. Other factors, as regularity of meals and time of eating meal, have significant effect in boys $(\mathrm{OR}=0.58, \mathrm{OR}=3.13$ and
$\mathrm{OR}=3.97)$ respectively. The combination of breakfast and school meal has a higher effect in boys than girls $(\mathrm{OR}=21.44$ and $\mathrm{OR}=5.73$ respectively).

Table 3. School Achievement for Boys, Girls and the Combined Group vs. some Social Factors.

\begin{tabular}{|c|c|c|c|c|c|c|c|c|c|c|c|c|}
\hline & \multicolumn{4}{|c|}{ Boys } & \multicolumn{4}{|l|}{ Girls } & \multicolumn{4}{|c|}{ Combined } \\
\hline & $\mathbf{n}$ & Failed $<\mathbf{5 0}$ & $\begin{array}{l}\text { Passed }> \\
50\end{array}$ & $X^{2}$ (sig) & $\mathbf{n}$ & Failed $<\mathbf{5 0}$ & $\begin{array}{l}\text { Passed }> \\
50\end{array}$ & $\mathrm{X}^{2}$ (sig) & $\mathbf{n}$ & Failed $<50$ & Passed $>50$ & $\mathrm{X}^{2}$ (sig) \\
\hline \multicolumn{13}{|l|}{ Child Order } \\
\hline Child $3^{\text {rd }}$ or more & 208 & $66(31.7 \%)$ & $\begin{array}{l}142 \\
(68.3 \%)\end{array}$ & \multirow{3}{*}{$\begin{array}{l}0.01 \\
(0.91)\end{array}$} & 202 & $\begin{array}{l}42 \\
(20.8 \%)\end{array}$ & $\begin{array}{l}160 \\
(79.2 \%)\end{array}$ & \multirow{3}{*}{$\begin{array}{l}0.33 \\
(0.56)\end{array}$} & 410 & $108(26.3 \%)$ & $\begin{array}{l}302 \\
(73.7 \%)\end{array}$ & \multirow{3}{*}{$\begin{array}{l}0.10 \\
(0.754)\end{array}$} \\
\hline \multirow[t]{2}{*}{ Child 1-2 } & 205 & $\begin{array}{l}64 \\
(31.2 \%)\end{array}$ & $\begin{array}{l}141 \\
(68.8 \%)\end{array}$ & & 194 & $\begin{array}{l}45 \\
(23.2 \%)\end{array}$ & $\begin{array}{l}149 \\
(76.8 \%)\end{array}$ & & 399 & $109(27.3 \%)$ & $\begin{array}{l}290 \\
(72.7 \%)\end{array}$ & \\
\hline & \multicolumn{3}{|c|}{$\mathrm{OR}=1.02(0.68-1.55)$} & & \multicolumn{3}{|c|}{$\mathrm{OR}=0.87(0.54-1.40)$} & & \multicolumn{3}{|c|}{$\mathrm{OR}=0.95(0.70-1.30)$} & \\
\hline \multicolumn{13}{|l|}{ Family Size } \\
\hline Large & 289 & $\begin{array}{l}85 \\
(29.4 \%)\end{array}$ & $\begin{array}{l}204 \\
(70.6 \%)\end{array}$ & \multirow{3}{*}{$\begin{array}{l}1.90 \\
(0.17)\end{array}$} & 282 & $\begin{array}{l}55 \\
(19.5 \%)\end{array}$ & $\begin{array}{l}227 \\
(80.5 \%)\end{array}$ & \multirow{3}{*}{$\begin{array}{l}3.58 \\
(0.06)\end{array}$} & 571 & $140(24.5 \%)$ & $\begin{array}{l}431 \\
(75.5 \%)\end{array}$ & \multirow{3}{*}{$\begin{array}{l}5.25 \\
(0.022)\end{array}$} \\
\hline Small & 124 & $\begin{array}{l}45 \\
(36.3 \%)\end{array}$ & $\begin{array}{l}79 \\
(63.7 \%)\end{array}$ & & 114 & $\begin{array}{l}32 \\
(28.1 \%)\end{array}$ & $\begin{array}{l}82 \\
(71.9 \%)\end{array}$ & & 238 & $\begin{array}{l}77 \\
(32.4 \%)\end{array}$ & $\begin{array}{l}161 \\
(67.6 \%)\end{array}$ & \\
\hline & \multicolumn{3}{|c|}{$\mathrm{OR}=0.73(0.47-1.14)$} & & \multicolumn{3}{|c|}{$\mathrm{OR}=0.62(0.38-1.03)$} & & \multicolumn{3}{|c|}{$\mathrm{OR}=0.68(0.49-0.95)$} & \\
\hline \multicolumn{13}{|c|}{ Mother Education (Low-High) } \\
\hline Low & 287 & $\begin{array}{l}83 \\
(28.9 \%)\end{array}$ & $\begin{array}{l}204 \\
(71.1 \%)\end{array}$ & \multirow{3}{*}{$\begin{array}{l}2.85 \\
(0.09)\end{array}$} & 281 & $\begin{array}{l}53 \\
(18.9 \%)\end{array}$ & $\begin{array}{l}228 \\
(81.1 \%)\end{array}$ & \multirow{3}{*}{$\begin{array}{l}5.45 \\
(0.019)\end{array}$} & 568 & $136(23.9 \%)$ & $\begin{array}{l}432 \\
(76.1 \%)\end{array}$ & \multirow{3}{*}{$\begin{array}{l}8.05 \\
(0.005)\end{array}$} \\
\hline High & 126 & $\begin{array}{l}47 \\
(37.3 \%)\end{array}$ & $\begin{array}{l}79 \\
(62.7 \%)\end{array}$ & & 115 & $\begin{array}{l}34 \\
(29.6 \%)\end{array}$ & $\begin{array}{l}81 \\
(70.4 \%)\end{array}$ & & 241 & $\begin{array}{l}81 \\
(33.6 \%)\end{array}$ & $\begin{array}{l}160 \\
(66.4 \%)\end{array}$ & \\
\hline & \multicolumn{3}{|c|}{$\mathrm{OR}=0.68(0.44-1.06)$} & & \multicolumn{3}{|c|}{$\mathrm{OR}=0.55(0.34-0.91)$} & & \multicolumn{2}{|c|}{$\mathrm{OR}=0.62(0.45-0.86)$} & & \\
\hline \multicolumn{13}{|c|}{ Father Education (Low-High) } \\
\hline Low & 255 & $\begin{array}{l}77 \\
(30.2 \%)\end{array}$ & $\begin{array}{l}178 \\
(69.8 \%)\end{array}$ & \multirow{3}{*}{$\begin{array}{l}0.51 \\
(0.48)\end{array}$} & 230 & $\begin{array}{l}46 \\
(20.0 \%)\end{array}$ & $\begin{array}{l}184 \\
(80.0 \%)\end{array}$ & \multirow{3}{*}{$\begin{array}{l}1.24 \\
(0.27)\end{array}$} & 485 & $123(25.4 \%)$ & $\begin{array}{l}362 \\
(74.6 \%)\end{array}$ & \multirow{3}{*}{$\begin{array}{l}1.32 \\
(0.251)\end{array}$} \\
\hline High & 158 & $\begin{array}{l}53 \\
(33.5 \%)\end{array}$ & $\begin{array}{l}105 \\
(66.5 \%)\end{array}$ & & 166 & $41(24.7 \%)$ & $\begin{array}{l}125 \\
(75.3 \%)\end{array}$ & & 324 & $\begin{array}{l}94 \\
(29.0 \%)\end{array}$ & $\begin{array}{l}230 \\
(71.0 \%)\end{array}$ & \\
\hline & $\mathrm{OR}=$ & $36(0.56-1.31$ & & & $\mathrm{OR}=$ & $76(0.47-1.23$ & & & $\mathrm{OR}=$ & $83(0.61-1.14)$ & & \\
\hline Father Job (Low-H & High) & & & & & & & & & & & \\
\hline Low & 242 & $\begin{array}{l}76 \\
(31.4 \%)\end{array}$ & $\begin{array}{l}166 \\
(68.6 \%)\end{array}$ & 0.001 & 247 & $\begin{array}{l}56 \\
(22.7 \%)\end{array}$ & $191(77.3 \%)$ & 0.19 & 489 & $132(27.0 \%)$ & $\begin{array}{l}357 \\
(73.0 \%)\end{array}$ & 0.02 \\
\hline High & 171 & $\begin{array}{l}54 \\
(31.6 \%)\end{array}$ & $\begin{array}{l}117 \\
(68.4 \%)\end{array}$ & $(0.97)$ & 149 & $\begin{array}{l}31 \\
(20.8 \%)\end{array}$ & $118(79.2 \%)$ & $(0.66)$ & 320 & $\begin{array}{l}85 \\
(26.6 \%)\end{array}$ & $\begin{array}{l}235 \\
(73.4 \%)\end{array}$ & $(0.892)$ \\
\hline & $\mathrm{OR}=$ & $9(0.65-1.51)$ & & & $\mathrm{OR}=$ & $12(0.68-1.83$ & & & $\mathrm{OR}=$ & $02(0.74-1.41)$ & & \\
\hline Mother Job (Low- & -High & & & & & & & & & & & \\
\hline Low & 358 & $\begin{array}{l}111 \\
(31.0 \%)\end{array}$ & $\begin{array}{l}247 \\
(69.0 \%)\end{array}$ & 0.28 & 341 & $\begin{array}{l}70 \\
(20.5 \%)\end{array}$ & $271(79.5 \%)$ & 2.98 & 699 & $181(25.9 \%)$ & $\begin{array}{l}518 \\
(74.1 \%)\end{array}$ & 2.26 \\
\hline High & 55 & $\begin{array}{l}19 \\
(34.5 \%)\end{array}$ & $\begin{array}{l}36 \\
(65.5 \%)\end{array}$ & $(0.60)$ & 55 & $\begin{array}{l}17 \\
(30.9 \%)\end{array}$ & $\begin{array}{l}38 \\
(69.1 \%)\end{array}$ & $(0.08)$ & 110 & $\begin{array}{l}36 \\
(32.7 \%)\end{array}$ & $\begin{array}{l}74 \\
(67.3 \%)\end{array}$ & $(0.133)$ \\
\hline & $\mathrm{OR}=$ & $35(0.47-1.55$ & & & $\mathrm{OR}=$ & $58(0.31-1.08$ & & & $\mathrm{OR}=$ & $72(0.47-1.11)$ & & \\
\hline
\end{tabular}

The majority of children who passed school (75.5\%) came from large families. Also, children with fathers which are illiterate or can only read and write and children with parents that are non-professionals or semi-professionals had passed school. There were no significant differences in these results. But children with illiterate or can only read and write mothers passed school with a significant difference than those with high educated mothers $(\mathrm{OR}=0.62)$.

When comparing the effect of these factors in boys and girls of the sample, we found that there are no significant differences in boys. But in girls, the mother's education has a significant difference (OR=0.55) (Table 3$)$.

The majority of children who had school meal passed math $(88.7 \%)$ verses $11.3 \%$ who failed. While children not having meal, only $21.9 \%$ passed and $78.1 \%$ failed $(\mathrm{OR}=28.05)$. But eating the meal in the morning has a high positive effect $(\mathrm{OR}=3.55)$ when comparing it with afternoon, or non-specific time $(\mathrm{OR}=5.16)$. And, similarly, those reported to consume breakfast had the same, but less obvious positive effect $(\mathrm{OR}=1.37), 60.9 \%$ of children having breakfast passed verses $39.1 \%$ failed).These results agree with work by Adrogué and Orlicki, 2013, who reported that eating breakfast, in contrast to fasting, may improve performance on the morning eaten. The long-term effects of eating breakfast on the performance of school children who do not have physical signs of severe undernourishment are less certain.

Having a mid-day snack has a positive effect $(\mathrm{OR}=2.54)$. The combined effect of breakfast and school meal has a high positive effect $(\mathrm{OR}=24.19)$, only $23.9 \%$ of children not having breakfast or a meal passed math. Dinner and snacking between meals had no significant effect on passing math (Table 4). Nutritional factors had increased chances of passing math. 
Table 4. Math Achievement for Boys, Girls and the Combined Group vs. some Nutritional Factors.

\begin{tabular}{|c|c|c|c|c|c|c|c|c|c|c|c|c|}
\hline & \multicolumn{4}{|c|}{ Boys } & \multicolumn{4}{|c|}{ Girls } & \multicolumn{4}{|c|}{ Combined } \\
\hline & $\mathrm{n}$ & Failed $<50$ & Passed $>50$ & $\mathbf{X}^{2}$ (sig) & n & Failed $<\mathbf{5 0}$ & Passed $>\mathbf{5 0}$ & $\mathrm{X}^{2}$ (sig) & $\mathbf{n}$ & Failed $<\mathbf{5 0}$ & Passed $>50$ & $X^{2}$ (sig) \\
\hline \multicolumn{13}{|l|}{ School Meal } \\
\hline No Meal & 120 & $102(85.0 \%)$ & $18(15.0 \%)$ & 197.13 & 120 & $86(71.7 \%)$ & $34(28.3 \%)$ & 174.04 & 233 & $182(78.1 \%)$ & $51(21.9 \%)$ & \multirow{3}{*}{$\begin{array}{l}349.30 \\
(0.000)\end{array}$} \\
\hline \multirow{2}{*}{ Meal } & 293 & $38(13.0 \%)$ & $255(87.0 \%)$ & $(0.000)$ & 276 & $21(7.6 \%)$ & $255(92.4 \%)$ & $(0.000)$ & 576 & $65(11.3 \%)$ & $511(88.7 \%)$ & \\
\hline & \multicolumn{4}{|c|}{$\mathrm{OR}=38.03(20.74-69.71)$} & \multicolumn{4}{|c|}{$\mathrm{OR}=30.71(16.92-55.76)$} & \multicolumn{3}{|c|}{$\mathrm{OR}=28.05(18.37-42.01)$} & \\
\hline \multicolumn{13}{|c|}{ Time of Distribution \& Eating School Meal } \\
\hline Afternoon & 32 & $5(15.6 \%)$ & $27(84.4 \%)$ & 3.25 & 24 & $1(4.2 \%)$ & $23(95.8 \%)$ & 2.66 & 56 & $6(10.7 \%)$ & $50(89.3 \%)$ & \multirow{3}{*}{$\begin{array}{l}5.73 \\
(0.02)\end{array}$} \\
\hline Morning & 63 & $3(4.8 \%)$ & $60(95.2 \%)$ & $(0.07)$ & 63 & 0 & $63(100.0 \%)$ & $(0.10)$ & 126 & $3(2.4 \%)$ & $123(97.6 \%)$ & \\
\hline & \multicolumn{4}{|c|}{$\mathrm{OR}=3.70(0.83-16.63)$} & \multicolumn{4}{|c|}{$\mathrm{OR}=$ Undefined } & \multicolumn{3}{|c|}{$\mathrm{OR}=4.92(1.18-20.44)$} & \\
\hline \multicolumn{13}{|c|}{ Time of Distribution \& Eating School Meal } \\
\hline Break & 152 & $22(14.5 \%)$ & $130(85.5 \%)$ & \multirow{2}{*}{$\begin{array}{l}4.09 \\
(0.04)\end{array}$} & 139 & $13(9.4 \%)$ & $126(90.6 \%)$ & \multirow{3}{*}{$\begin{array}{l}6.30 \\
(0.01)\end{array}$} & 291 & $35(12.0 \%)$ & $256(88.0 \%)$ & \multirow{2}{*}{$\begin{array}{l}9.88 \\
(0.001)\end{array}$} \\
\hline Morning & 63 & $3(4.8 \%)$ & $60(95.2 \%)$ & & 63 & 0 & $63(100.0 \%)$ & & 126 & $3(2.4 \%)$ & $123(97.6 \%)$ & \\
\hline & $\mathrm{OR}=$ & $3.38(0.98-11$ & & & $\mathrm{OR}=$ & Undefined & & & $\mathrm{OR}=$ & $5.61(1.69-18.5$ & & \\
\hline Time of Distribu & tion \& & Eating School & Meal & & & & & & & & & \\
\hline $\begin{array}{l}\text { No Specific } \\
\text { Time }\end{array}$ & 41 & $8(19.5 \%)$ & $33(80.5 \%)$ & 5.71 & 47 & $7(14.9 \%)$ & $40(85.1 \%)$ & 10.02 & 88 & $15(17.0 \%)$ & $73(83.0 \%)$ & 14.46 \\
\hline Morning & 63 & $3(4.8 \%)$ & $60(95.2 \%)$ & & 63 & 0 & $63(100.0 \%)$ & & 126 & $3(2.4 \%)$ & $123(97.6 \%)$ & \\
\hline & $\mathrm{OR}=$ & $4.85(1.20-19$ & & & $\mathrm{OR}=$ & Undefined & & & $\mathrm{OR}=$ & $8.42(2.36-30.0$ & & \\
\hline Breakfast & & & & & & & & & & & & \\
\hline No & 111 & $40(36.0 \%)$ & $71(64.0 \%)$ & 0.31 & 141 & $44(31.2 \%)$ & $97(68.8 \%)$ & 1.95 & 252 & $118(46.8 \%)$ & $134(53.2 \%)$ & 4.22 \\
\hline Yes & 302 & $100(33.1 \%)$ & $202(66.9 \%)$ & $(0.58)$ & 255 & $63(24.7 \%)$ & $192(75.3 \%)$ & $(0.16)$ & 557 & $218(39.1 \%)$ & $339(60.9 \%)$ & $(0.024)$ \\
\hline & $\mathrm{OR}=$ & $1.38(0.72-1.7$ & & & $\mathrm{OR}=$ & $1.38(0.88-2$. & & & $\mathrm{OR}=$ & $1.37(1.01-1.85$ & & \\
\hline Breakfast Before & Scho & & & & & & & & & & & \\
\hline No & 109 & $38(34.9 \%)$ & $71(65.1 \%)$ & 0.06 & 152 & $53(34.9 \%)$ & $99(65.1 \%)$ & 7.71 & 261 & $122(46.7 \%)$ & $139(53.3 \%)$ & 4.31 \\
\hline Yes & 304 & $102(33.6 \%)$ & $202(66.4 \%)$ & $(0.80)$ & 244 & $54(22.1 \%)$ & $190(77.9 \%)$ & $(0.005)$ & 548 & $214(39.1 \%)$ & $334(60.9 \%)$ & $(0.023)$ \\
\hline & $\mathrm{OR}=$ & $1.06(0.67-1.6$ & & & $\mathrm{OR}=$ & $1.88(1.20-2$. & & & $\mathrm{OR}=$ & $1.37(1.025-1.8$ & & \\
\hline School Meal \& I & Breakf & & & & & & & & & & & \\
\hline $\begin{array}{l}\text { No School Meal } \\
\& \text { No Breakfast }\end{array}$ & 40 & $31(77.5 \%)$ & $9(22.5 \%)$ & 76.54 & 48 & $36(75.0 \%)$ & $12(25.0 \%)$ & 118.41 & 88 & $67(76.1 \%)$ & $21(23.9 \%)$ & 170.26 \\
\hline $\begin{array}{l}\text { School Meal\& } \\
\text { Breakfast }\end{array}$ & 224 & $31(13.8 \%)$ & $193(86.2 \%)$ & $(0.000)$ & 188 & $17(9.0 \%)$ & $171(91.0 \%)$ & $(0.000)$ & 412 & $48(11.7 \%)$ & $364(88.3 \%)$ & \\
\hline & $\mathrm{OR}=$ & 21.44 & & & $\mathrm{OR}=$ & 40.24 (17.98 & $-90.03)$ & & $\mathrm{OR}=$ & $24.19(13.61-4$ & $3.01)$ & \\
\hline $\begin{array}{l}\text { Number of } \\
\text { Meals }\end{array}$ & & & & & & & & & & & & \\
\hline $\begin{array}{l}\text { Less than } 3 \\
\text { meals/day }\end{array}$ & 41 & $10(24.4 \%)$ & $31(75.6 \%)$ & 1.84 & 63 & $19(30.2 \%)$ & $44(69.8 \%)$ & 0.37 & 104 & $64(61.5 \%)$ & $40(38.5 \%)$ & 14.03 \\
\hline $\begin{array}{l}3 \text { or more } \\
\text { meals/day }\end{array}$ & 372 & $130(34.9 \%)$ & $242(65.1 \%)$ & $(0.18)$ & 333 & $88(26.4 \%)$ & $245(73.6 \%)$ & $(0.54)$ & 705 & $296(42.0 \%)$ & $409(58.0 \%)$ & $(0.000)$ \\
\hline & $\mathrm{OR}=$ & $0.60(0.28-1.26$ & & & $\mathrm{OR}=$ & $1.20(0.67-2$. & & & $\mathrm{OR}=$ & $2.21(1.45-3.3)$ & & \\
\hline Snacks Between & Meals & & & & & & & & & & & \\
\hline No & 88 & $28(31.8 \%)$ & $60(68.2 \%)$ & 0.22 & 60 & $9(15.0 \%)$ & $51(8$ & 5.18 & 148 & $56(37.8 \%)$ & & 1.02 \\
\hline Yes & 325 & $112(34.5 \%)$ & $213(65.5 \%)$ & $(0.64)$ & 336 & $98(29.2 \%)$ & $238(70.8 \%)$ & $(0.02)$ & 661 & $280(42.4 \%)$ & $381(57.6 \%)$ & $(0.18)$ \\
\hline & $\mathrm{OR}=$ & $0.89(0.54-1.4$ & & & $\mathrm{OR}=$ & $0.43(0.20-0.9$ & & & $\mathrm{OR}=$ & $0.83(057-1.19$ & & \\
\hline Mid Day Snack & & & & & & & & & & & & \\
\hline No & 60 & $18(30.0 \%)$ & $42(70.0 \%)$ & 0.48 & 35 & $15(42.9 \%)$ & $20(57.1 \%)$ & 4.88 & 97 & $60(63.2 \%)$ & $37(36.8 \%)$ & 3.58 \\
\hline Yes & 353 & $122(34.6 \%)$ & $231(65.4 \%)$ & $(0.49)$ & 361 & $92(25.5 \%)$ & $269(74.5 \%)$ & $(0.03)$ & 714 & $288(40.3 \%)$ & $426(59.7 \%)$ & $(0.038)$ \\
\hline & $\mathrm{OR}=$ & $0.81(0.45-1.47$ & & & $\mathrm{OR}=$ & $2.19(1.08-4$. & & & $\mathrm{OR}=$ & $2.54(1.63-3.95$ & & \\
\hline Meals on Time & Regul & ar Meals) & & & & & & & & & & \\
\hline No & 123 & $32(26.0 \%)$ & $91(74.0 \%)$ & 4.86 & 132 & $34(25.8 \%)$ & $98(74.2 \%)$ & 0.16 & 554 & $341(61.6 \%)$ & $213(38.4 \%)$ & 41.49 \\
\hline Yes & 290 & $108(37.2 \%)$ & $182(62.8 \%)$ & $(0.03)$ & 264 & $73(27.7 \%)$ & $191(72.3 \%)$ & $(0.69)$ & 255 & $95(37.3 \%)$ & $160(62.7 \%)$ & $(0.000)$ \\
\hline & $\mathrm{OR}=$ & $0.59(0.37-0.9$ & & & $\mathrm{OR}=$ & $0.91(0.56-1$. & & & $\mathrm{OR}=$ & $2.70(1.96-3.60$ & & \\
\hline Dinner & & & & & & & & & & & & \\
\hline No & 21 & $7(33.3 \%)$ & $14(66.7 \%)$ & 0.004 & 41 & $15(36.6 \%)$ & $26(63.4 \%)$ & 2.12 & 62 & $27(43.5 \%)$ & $35(56.5)$ & 0.107 \\
\hline Yes & 391 & $133(34.0 \%)$ & $258(66.0 \%)$ & $(0.95)$ & 355 & $92(25.9 \%)$ & $263(74.1 \%)$ & $(0.15)$ & 746 & $309(41.4 \%)$ & $437(58.6 \%)$ & $(0.421)$ \\
\hline & $\mathrm{OR}=$ & $0.97(0.38-2.46$ & & & $\mathrm{OR}=$ & $1.65(0.84-3$. & & & $\mathrm{OR}=$ & $1.09(0.65-1.84$ & & \\
\hline
\end{tabular}

When comparing the effect of nutritional factors in boys and girls in the sample, there is a difference in factors affecting the passing math. In girls, having a meal had a significant effect $(\mathrm{OR}=30.71)$; eating the meal $(\mathrm{OR}=3.48)$. Having the school meal in the morning had positive significant effect on passing math compared with the afternoon an on no specific time respectively. Also, having breakfast before school $(\mathrm{OR}=1.88)$, the mid-day snack has a significant effect $(\mathrm{OR}=2.19)$ and the combination of breakfast and school meal $(\mathrm{OR}=40.24)$.

As for boys, having a meal $(\mathrm{OR}=38.03)$; has a significant effect which is lower compared with girls. Having the school 
meal in the morning had positive significant effect on passing math compared with the afternoon an on no specific time $(\mathrm{OR}=3.70$ and $\mathrm{OR}=4.85)$ respectively. Other factors, as regularity of meals have slight significant effect in boys
$(\mathrm{OR}=0.59)$. The combination of breakfast and school meal has a lower effect in boys than girls $(\mathrm{OR}=21.44$ and $\mathrm{OR}=40.24$ respectively).

Table 5. Math Achievement for Boys, Girls and the Combined Group vs. some Social Factors.

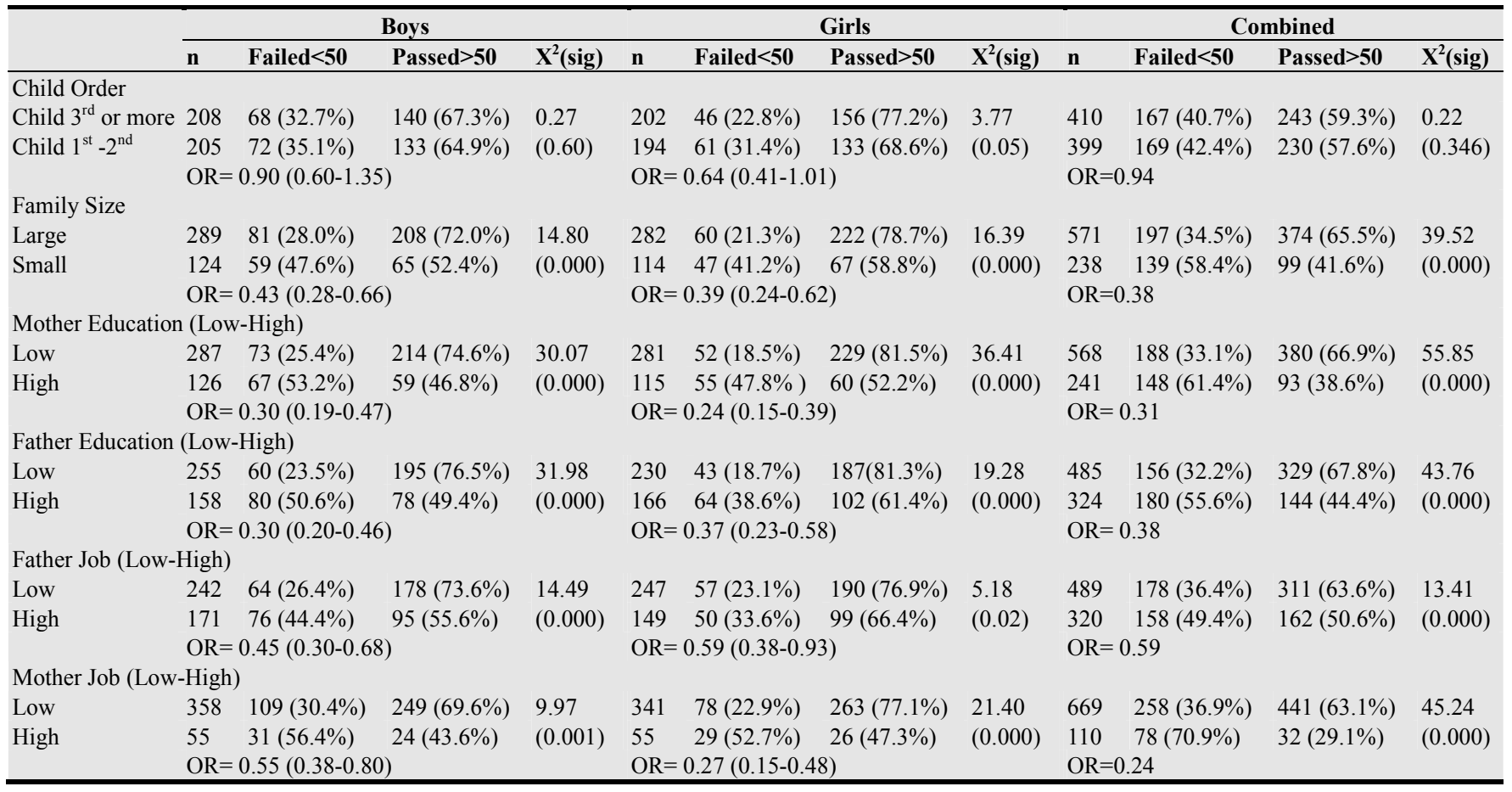

More than half $(65.5 \%)$ of children who passed math came from large families $(\mathrm{OR}=0.38)$. Also, children with parents which are illiterate or can only read and write and children with parents that are non-professionals or semi-professionals had passed math, with significant differences in these results $(\mathrm{OR}=0.31, \mathrm{OR}=0.38, \mathrm{OR}=0.59$ and $\mathrm{OR}=0.24$ respectively).

When comparing the effect of these factors in boys and girls of the sample, we found that there are significant differences in boys $(\mathrm{OR}=0.30, \mathrm{OR}=0.30, \mathrm{OR}=0.45$ and $\mathrm{OR}=$ 0.55 respectively). But in girls, the child's order of birth, also, has a significant difference $(\mathrm{OR}=0.24, \mathrm{OR}=0.37, \mathrm{OR}=0.59$ and $\mathrm{OR}=0.27$ respectively) (Table 5).

The average school grades for the sample was $62.14 \pm 17.55 ; 64.76 \pm 17.82$ for girls and $59.63 \pm 17.73$ for boys.

Table 6, represents the average school grades for the children receiving, and not receiving, the school meal, in combination with different nutritional factors. It can be noticed that the children receiving a school meal had significantly higher grades regardless the other factor (breakfast, number of meals, dinner.....). Our results agree with work by Adrogué and Orlicki, 2013 who reported that the long-term effects of eating breakfast on the performance of school children who do not have physical signs of severe undernourishment are less certain. The regularity of meals had significant positive effect on grades regardless the effect of school meal. Also the mid-day snack had significant positive effect but only in case of children receiving the meal. Our results agree with previous work by Acham et al., 2012, who reported that the midday meal had a stronger effect on achievements compared to breakfast. They also reported that the combination of midday meal and breakfast had higher effect than only one type of meal.

Table 6. School Achievement Scores for Boys, Girls and the Combined Group vs. School Meal and some Nutritional Factors.

\begin{tabular}{|c|c|c|c|c|c|c|c|c|c|c|}
\hline & \multicolumn{5}{|c|}{ Boys } & \multicolumn{5}{|c|}{ Girls } \\
\hline & \multicolumn{2}{|c|}{ Not Having Meal } & \multicolumn{2}{|c|}{ Having Meal } & \multirow{2}{*}{$-t(\mathbf{p})$} & \multicolumn{2}{|c|}{ Not Having Meal } & \multicolumn{2}{|c|}{ Having Meal } & \multirow{2}{*}{$-t(\mathbf{p})$} \\
\hline & $\bar{n}$ & Mean \pm SD & n & Mean \pm SD & & $\bar{n}$ & Mean \pm SD & n & Mean \pm SD & \\
\hline \multicolumn{11}{|c|}{ Breakfast } \\
\hline No & 40 & $48.18 \pm 11.11$ & 71 & $63.69 \pm 17.34$ & $-4.70(0.000)$ & 48 & $51.70 \pm 11.71$ & 93 & $69.34 \pm 16.90$ & $-6.47(0.000)$ \\
\hline Yes & 78 & $48.66 \pm 9.35$ & 224 & $64.28 \pm 19.97$ & $-7.45(0.000)$ & 67 & $51.13 \pm 9.74$ & 188 & $70.68 \pm 17.30$ & $-8.76(0.000)$ \\
\hline$t(\mathrm{p})$ & & $-0.25(0.80)$ & & $0.18(0.85)$ & & & $0.28(0.78)$ & & $-0.61(0.54)$ & \\
\hline \multicolumn{11}{|c|}{ Breakfast Before School } \\
\hline No & 39 & $47.19 \pm 11.39$ & 70 & $64.23 \pm 19.23$ & $-5.06(0.000)$ & 55 & $51.05 \pm 11.11$ & 97 & $69.53 \pm 17.65$ & $-7.01(0.000)$ \\
\hline Yes & 79 & $49.15 \pm 9.14$ & 225 & $64.04 \pm 17.61$ & $-7.18(0.000)$ & 60 & $51.65 \pm 10.12$ & 184 & $70.61 \pm 16.92$ & $-8.20(0.000)$ \\
\hline$t(\mathrm{p})$ & & $-1.00(0.32)$ & & $0.08(0.94)$ & & & $-0.30(0.76)$ & & $-0.50(0.62)$ & \\
\hline Num & er da & & & & & & & & & \\
\hline
\end{tabular}




\begin{tabular}{|c|c|c|c|c|c|c|c|c|c|c|}
\hline & \multicolumn{5}{|c|}{ Boys } & \multicolumn{5}{|c|}{ Girls } \\
\hline & \multicolumn{2}{|c|}{ Not Having Meal } & \multicolumn{2}{|c|}{ Having Meal } & \multirow{2}{*}{$t(\mathbf{p})$} & \multicolumn{2}{|c|}{ Not Having Meal } & \multicolumn{2}{|c|}{ Having Meal } & \multirow{2}{*}{$t(\mathbf{p})$} \\
\hline & n & Mean \pm SD & n & Mean \pm SD & & $\mathrm{n}$ & Mean \pm SD & n & Mean \pm SD & \\
\hline $\begin{array}{l}\text { Less than } 3 \\
\text { meals/day }\end{array}$ & 12 & $47.31 \pm 8.67$ & 29 & $63.56 \pm 18.10$ & $-4.32(0.000)$ & 16 & $48.83 \pm 14.20$ & 47 & $66.89 \pm 18.44$ & $-3.57(0.001)$ \\
\hline $\begin{array}{l}3 \text { or more } \\
\text { meals/day }\end{array}$ & 106 & $48.63 \pm 10.10$ & 266 & $68.48 \pm 16.27$ & $-8.01(0.000)$ & 99 & $51.78 \pm 9.88$ & 234 & $70.91 \pm 16.84$ & $-10.55(0.000)$ \\
\hline$t(\mathrm{p})$ & & $-0.44(0.66)$ & & $-1.51(0.13)$ & & & $-1.04(0.30)$ & & $-1.47(0.14)$ & \\
\hline No & & & & & & & & & & \\
\hline Yes & 52 & $4.90 \pm 9.05$ & 91 & $61.94 \pm 1 / .91$ & $-5.81(0.000)$ & 42 & $49.93 \pm 10.61$ & 90 & $70.69 \pm 17.09$ & $-5.91(0.000)$ \\
\hline$t(\mathrm{p})$ & & $-0.44(0.66)$ & 204 & $\begin{array}{l}0.00117 .11 \\
-3.11(0.002)\end{array}$ & & 73 & $\begin{array}{l}53.86 \pm 10.12 \\
-1.94(0.05)\end{array}$ & 91 & $\begin{array}{l}70.02 \pm 17.22 \\
0.30(0.76)\end{array}$ & \\
\hline Dinner & & & & & & & & & & \\
\hline No & 7 & $48.25 \pm 5.82$ & 14 & $63.73 \pm 18.06$ & $-3.77(0.001)$ & 14 & $49.07 \pm 14.18$ & 27 & $69.11 \pm 13.42$ & $-4.45(0.000)$ \\
\hline Yes & 111 & $48.52 \pm 10.16$ & 280 & $71.46 \pm 15.60$ & $-8.36(0.000)$ & 101 & $51.69 \pm 10.01$ & 254 & $70.35 \pm 17.52$ & $-10.07(0.000)$ \\
\hline$t(\mathrm{p})$ & & $-0.07(0.95)$ & & $-1.57(0.12)$ & & & $-0.87(0.39)$ & & $-0.36(0.72)$ & \\
\hline Mid-Day Snack & & & & & & & & & & \\
\hline No & 17 & $47.26 \pm 10.47$ & 43 & $62.62 \pm 18.15$ & $-3.27(0.002)$ & 17 & $50.85 \pm 10.51$ & 18 & $58.43 \pm 16.80$ & $-1.59(0.12)$ \\
\hline Yes & 101 & $48.71 \pm 9.88$ & 252 & $64.33 \pm 17.97$ & $-8.25(0.000)$ & 98 & $51.46 \pm 10.62$ & 263 & $71.04 \pm 16.91$ & $-10.70(0.000)$ \\
\hline$t(\mathrm{p})$ & & $-0.55(0.58)$ & & $-0.58(0.57)$ & & & $-0.22(0.83)$ & & $-3.06(0.002)$ & \\
\hline
\end{tabular}

Table 6. Continued.

\begin{tabular}{|c|c|c|c|c|c|}
\hline & \multicolumn{5}{|c|}{ Combined } \\
\hline & \multicolumn{2}{|c|}{ Not Having Meal } & \multicolumn{2}{|c|}{ Having Meal } & \multirow{2}{*}{$t(\mathbf{p})$} \\
\hline & $\mathrm{n}$ & Mean \pm SD & $\mathrm{n}$ & Mean \pm SD & \\
\hline \multicolumn{6}{|l|}{ Breakfast } \\
\hline No & 88 & $49.80 \pm 9.58$ & 164 & $67.15 \pm 18.41$ & $-7.90(0.000)$ \\
\hline Yes & 145 & $50.10 \pm 11.51$ & 412 & $67.06 \pm 17.62$ & $-11.22(0.000)$ \\
\hline$t(\mathrm{p})$ & & $-0.21(0.83)$ & & $0.06(0.96)$ & \\
\hline \multicolumn{6}{|l|}{ Breakfast Before School } \\
\hline No & 94 & $49.45 \pm 11.33$ & 167 & $66.99 \pm 17.59$ & $-8.52(0.000)$ \\
\hline Yes & 139 & $50.23 \pm 9.62$ & 409 & $67.31 \pm 18.46$ & $-10.70(0.000)$ \\
\hline$t(\mathrm{p})$ & & $-0.56(0.58)$ & & $-0.19(0.85)$ & \\
\hline \multicolumn{6}{|c|}{ Number of Meals per day } \\
\hline Less than 3 meals/day & 28 & $48.18 \pm 11.97$ & 76 & $67.64 \pm 17.56$ & $-5.41(0.000)$ \\
\hline 3 or more meals/day & 205 & $50.15 \pm 10.09$ & 500 & $67.00 \pm 17.89$ & $-12.68(0.000)$ \\
\hline$t(\mathrm{p})$ & & $-0.95(0.34)$ & & $0.29(0.77)$ & \\
\hline \multicolumn{6}{|l|}{ Meals on Time } \\
\hline No & 74 & $48.86 \pm 9.96$ & 181 & $65.85 \pm 18.05$ & $-8.22(0.000)$ \\
\hline Yes & 159 & $52.17 \pm 10.81$ & 395 & $69.79 \pm 17.08$ & $-11.19(0.000)$ \\
\hline$t(\mathrm{p})$ & & $-2.30(0.02)$ & & $-2.47(0.014)$ & \\
\hline \multicolumn{6}{|l|}{ Dinner } \\
\hline No & 21 & $48.80 \pm 11.87$ & 41 & $66.88 \pm 18.09$ & $-5.89(0.000)$ \\
\hline Yes & 212 & $50.03 \pm 10.19$ & 534 & $69.91 \pm 14.06$ & $-12.78(0.000)$ \\
\hline$t(\mathrm{p})$ & & $-0.52(0.60)$ & & $-1.05(0.29)$ & \\
\hline \multicolumn{6}{|l|}{ Mid-Day Snack } \\
\hline No & 34 & $49.06 \pm 10.49$ & 61 & $61.39 \pm 17.73$ & $-3.70(0.000)$ \\
\hline Yes & 199 & $50.06 \pm 10.32$ & 515 & $67.76 \pm 17.74$ & $-13.23(0.000)$ \\
\hline$t(\mathrm{p})$ & & $-0.52(0.60)$ & & $-2.65(0.008)$ & \\
\hline
\end{tabular}

As for boys, the average school grades were not significantly affected by nutritional factors, except in case of the regularity of meals in combination with school meal, which had a significant positive effect. The same is true for girls, where the regularity of meals alone had a significant positive effect and the mid-day snack in combination with school meal had a significant positive effect. It can be noticed that, both boy and girls, receiving a school meal had significantly higher grades than those not a school meal regardless the other factor.

When considering the effect of social factors we found that they had no significant effect on school grades of the children (the sample or in boy and girls separately) not receiving school meal. But when comparing the effect of each of these factors on children receiving school meal, we found a significant positive effect on school grades for the children of the sample. Our results agree with previous work done by

As for boys, we found a significant positive effect for all factors except for order of birth and mother's job for children receiving school meal. But for girls receiving the school meal, only the parent education and father's job showed a significant positive effect, while other factors showed non-significant effects (Table 7). In general girls scored higher than boys, these results are in contrast with work by Kamogawa, 2003 and Hamid Jan et al., 2011 who reported that female Malaysian students earned better grades than males. 
Table 7. School Achievement Scores for Boys, Girls and the Combined Group vs. School Meal and some Social Factors.

\begin{tabular}{|c|c|c|c|c|c|c|c|c|c|c|}
\hline & \multicolumn{5}{|c|}{ Boys } & \multicolumn{5}{|c|}{ Girls } \\
\hline & \multicolumn{2}{|c|}{ Not Having Meal } & \multicolumn{2}{|c|}{ Having Meal } & \multirow{2}{*}{$\begin{array}{l}t \\
\mathbf{p}\end{array}$} & \multicolumn{2}{|c|}{ Not Having Meal } & \multicolumn{2}{|c|}{ Having Meal } & \multirow{2}{*}{$\begin{array}{r}t \\
-p\end{array}$} \\
\hline & n & Mean \pm SD & $\mathbf{n}$ & Mean \pm SD & & $\mathrm{n}$ & Mean \pm SD & $\mathbf{n}$ & Mean \pm SD & \\
\hline \multicolumn{11}{|c|}{ Child Order of Birth } \\
\hline Child $3^{\text {rd }}$ or more & 49 & $48.94 \pm 10.14$ & 159 & $62.35 \pm 18.67$ & $-4.81(0.000)$ & 51 & $51.81 \pm 10.85$ & 151 & $68.73 \pm 16.77$ & $-6.74(0.000)$ \\
\hline Child $1^{\text {st }}-2^{\text {nd }}$ & 69 & $48.19 \pm 9.86$ & 136 & $66.11 \pm 16.97$ & $-8.10(0.000)$ & 64 & $51.01 \pm 10.40$ & 130 & $71.98 \pm 17.49$ & $-8.85(0.000)$ \\
\hline$t(\mathrm{p})$ & & $0.40(0.69)$ & & $-1.79(0.07)$ & & & $0.40(0.69)$ & & $-1.59(0.11)$ & \\
\hline \multicolumn{11}{|l|}{ Family Size } \\
\hline Large & 56 & $48.62 \pm 10.37$ & 233 & $62.95 \pm 18.22$ & $-5.66(0.000)$ & 53 & $50.57 \pm 10.69$ & 229 & $69.86 \pm 17.06$ & $-7.87(0.000)$ \\
\hline Small & 62 & $48.40 \pm 9.61$ & 62 & $68.36 \pm 16.45$ & $-8.25(0.000)$ & 62 & $52.05 \pm 10.49$ & 52 & $71.89 \pm 17.62$ & $-7.44(0.000)$ \\
\hline$t(\mathrm{p})$ & & $0.12(0.91)$ & & $-2.12(0.04)$ & & & $-0.75(0.46)$ & & $-0.77(0.44)$ & \\
\hline \multicolumn{11}{|c|}{ Mother Education (Low-High) } \\
\hline Low & 47 & $47.89 \pm 9.78$ & 240 & $62.59 \pm 17.88$ & $-5.47(0.000)$ & 52 & $52.25 \pm 11.17$ & 229 & $68.78 \pm 16.97$ & $-6.70(0.000)$ \\
\hline High & 71 & $48.90 \pm 10.09$ & 55 & $70.61 \pm 17.40$ & $-8.91(0.000)$ & 63 & $50.64 \pm 10.06$ & 52 & $76.65 \pm 16.62$ & $-10.35(0.000)$ \\
\hline$t(\mathrm{p})$ & & $-0.54(0.59)$ & & $-3.03(0.003)$ & & & $0.81(0.42)$ & & $0.68(0.003)$ & \\
\hline \multicolumn{11}{|c|}{ Father Education (Low-High) } \\
\hline Low & 35 & $46.36 \pm 10.79$ & 220 & $61.71 \pm 18.05$ & $-4.89(0.000)$ & 38 & $52.06 \pm 11.16$ & 192 & $68.15 \pm 17.12$ & $-5.56(0.000)$ \\
\hline High & 83 & $49.40 \pm 9.48$ & 75 & $71.05 \pm 15.89$ & $-10.52(0.000)$ & 77 & $51.03 \pm 10.31$ & 89 & $74.72 \pm 16.43$ & $-10.93(0.000)$ \\
\hline$t(\mathrm{p})$ & & $-1.53(0.13)$ & & $-3.98(0.000)$ & & & $0.49(0.62)$ & & $-3.03(0.003)$ & \\
\hline \multicolumn{11}{|c|}{ Father Job (Low-High) } \\
\hline Low & 47 & $46.34 \pm 9.75$ & 195 & $62.11 \pm 17.63$ & $-5.91(0.000)$ & 51 & $50.88 \pm 11.21$ & 196 & $67.20 \pm 17.14$ & $-6.44(0.000)$ \\
\hline High & 71 & $49.93 \pm 9.87$ & 100 & $67.93 \pm 18.09$ & $-7.62(0.000)$ & 64 & $51.75 \pm 10.08$ & 85 & $77.24 \pm 15.07$ & $-11.69(0.000)$ \\
\hline$t(\mathrm{p})$ & & $-1.94(0.05)$ & & $-2.66(0.008)$ & & & $-0.44(0.66)$ & & $-4.67(0.000)$ & \\
\hline \multicolumn{11}{|c|}{ Mother Job (Low-High) } \\
\hline Low & 82 & $47.79 \pm 9.36$ & 276 & $63.60 \pm 17.91$ & $-7.68(0.000)$ & 78 & $51.46 \pm 11.41$ & 263 & $69.76 \pm 17.13$ & $-8.87(0.000)$ \\
\hline High & 36 & $50.10 \pm 11.11$ & 19 & $71.07 \pm 17.98$ & $-5.34(0.000)$ & 37 & $51.18 \pm 8.63$ & 18 & $77.17 \pm 16.43$ & $-7.72(0.000)$ \\
\hline$t(\mathrm{p})$ & & $-1.16(0.25)$ & & $-1.76(0.08)$ & & & $0.13(0.89)$ & & $-1.78(0.08)$ & \\
\hline
\end{tabular}

Table 7. Continued.

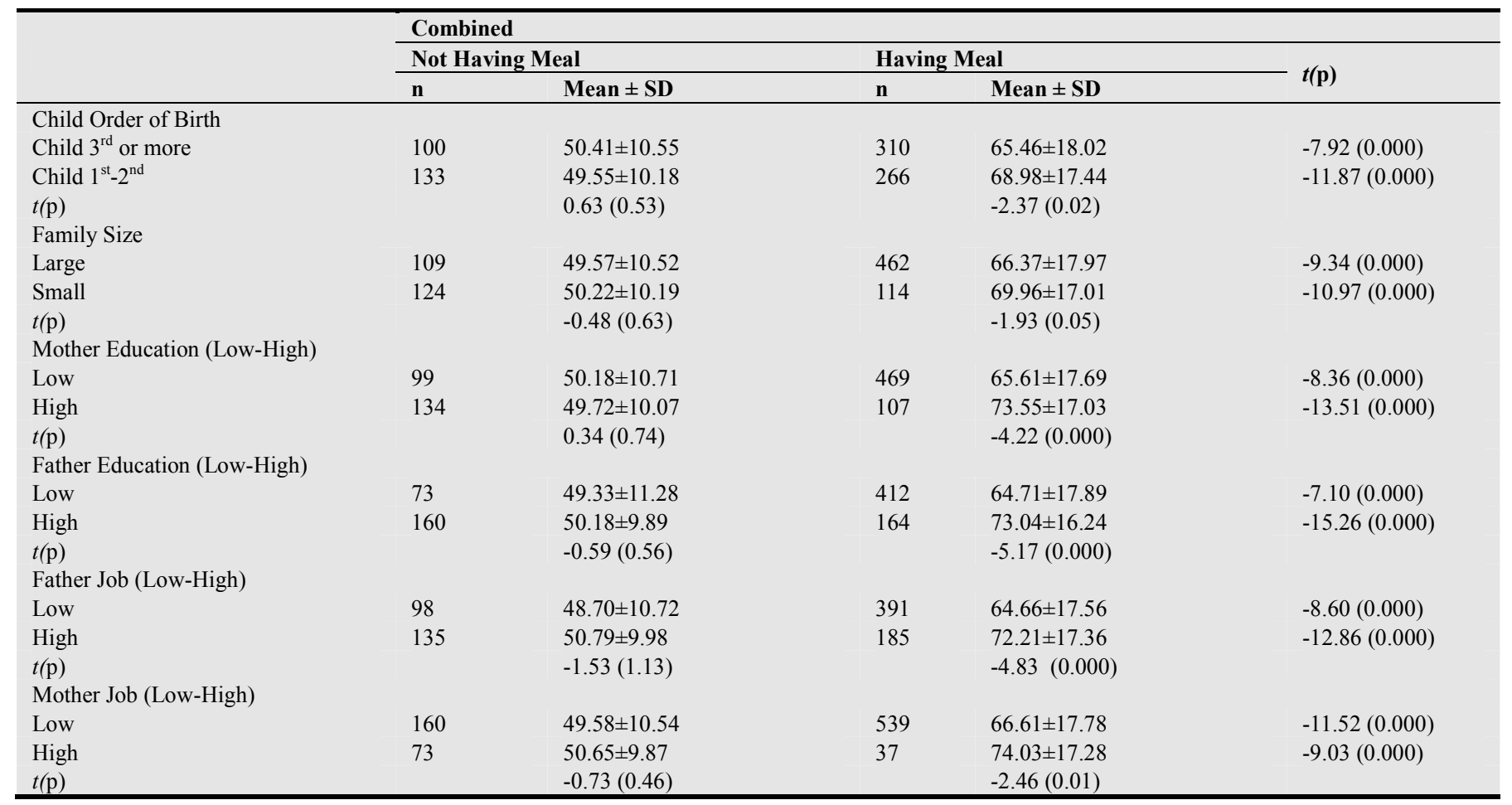

When comparing math grade with regard to nutritional factors, for children not receiving school meal, it was found that the regularity of meals only had a statistically significant positive effect on math grades. While for children receiving school meal, it was found that the regularity of meals and the midday snack had a statistically significant positive effect on math grades.

While for boys, having the breakfast and school meal or meal regularity with school meal had a positive significant effect on math grades. As for girls, having a mid-day snack with the school meal had a statistically positive significant effect on math grades (Table 8). 
Table 8. Math Test School Achievement Scores for Boys, Girls and the Combined Group vs. School Meal and some Nutritional Factors.

\begin{tabular}{|c|c|c|c|c|c|c|c|c|c|c|}
\hline & \multicolumn{5}{|c|}{ Boys } & \multicolumn{5}{|c|}{ Girls } \\
\hline & \multicolumn{2}{|c|}{ Not Having Meal } & \multicolumn{2}{|c|}{ Having Meal } & \multirow{2}{*}{$\begin{array}{l}t \\
\mathrm{p}\end{array}$} & \multicolumn{2}{|c|}{ Not Having Meal } & \multicolumn{2}{|c|}{ Having Meal } & \multirow{2}{*}{$\begin{array}{l}t \\
\mathbf{p}\end{array}$} \\
\hline & $\mathrm{n}$ & Mean \pm SD & n & Mean \pm SD & & $\mathrm{n}$ & Mean \pm SD & n & Mean \pm SD & \\
\hline \multicolumn{11}{|l|}{ Breakfast } \\
\hline No & 40 & $24.56 \pm 14.32$ & 159 & $60.63 \pm 21.09$ & $-16.44(0.000)$ & 48 & $27.86 \pm 19.12$ & 93 & $68.97 \pm 19.76$ & $-11.83(0.000)$ \\
\hline Yes & 78 & $27.17 \pm 16.02$ & 136 & $64.19 \pm 17.72$ & $-9.42(0.000)$ & 67 & $29.89 \pm 18.30$ & 188 & $69.66 \pm 19.63$ & $-14.49(0.000)$ \\
\hline$t(\mathrm{p})$ & & $-1.14(0.26)$ & & $-3.02(0.003)$ & & & $-0.57(0.57)$ & & $-0.28(0.78)$ & \\
\hline \multicolumn{11}{|l|}{ Breakfast Before School } \\
\hline No & 39 & $24.78 \pm 16.27$ & 70 & $63.64 \pm 20.09$ & $-10.34(0.000)$ & 55 & $26.18 \pm 19.21$ & 97 & $68.81 \pm 21.19$ & $-12.32(0.000)$ \\
\hline Yes & 79 & $26.54 \pm 16.28$ & 225 & $64.35 \pm 19.32$ & $-15.82(0.000)$ & 60 & $31.67 \pm 17.77$ & 184 & $69.76 \pm 18.82$ & $-13.80(0.000)$ \\
\hline$t(\mathrm{p})$ & & $-0.60(0.55)$ & & $0.26(0.80)$ & & & $-1.59(0.11)$ & & $-0.39(0.70)$ & \\
\hline \multicolumn{11}{|c|}{ Number of Meals per day } \\
\hline Less than 3 meals/day & 12 & $25.00 \pm 15.15$ & 29 & $63.42 \pm 20.27$ & $-7.99(0.000)$ & 16 & $30.31 \pm 19.47$ & 47 & $66.47 \pm 18.68$ & $-6.62(0.000)$ \\
\hline 3 or more meals/day & 106 & $25.40 \pm 15.05$ & 266 & $67.41 \pm 15.59$ & $-17.48(0.000)$ & 99 & $28.84 \pm 18.54$ & 234 & $70.03 \pm 19.81$ & $-17.67(0.000)$ \\
\hline$t(\mathrm{p})$ & & $-0.09(0.93)$ & & $-1.03(0.31)$ & & & $0.29(0.77)$ & & $-1.14(0.26)$ & \\
\hline \multicolumn{11}{|l|}{ Meals on Time } \\
\hline No & 32 & $23.20 \pm 14.47$ & 91 & $61.41 \pm 20.16$ & $-10.60(0.000)$ & 42 & $30.83 \pm 15.81$ & 90 & $71.29 \pm 17.99$ & $-5.53(0.000)$ \\
\hline Yes & 86 & $31.17 \pm 15.07$ & 204 & $69.20 \pm 18.20$ & $-15.93(0.000)$ & 73 & $28.01 \pm 20.05$ & 191 & $68.56 \pm 20.35$ & $-18.01(0.000)$ \\
\hline$t(\mathrm{p})$ & & $-2.63(0.01)$ & & $-3.16(0.002)$ & & & $0.78(0.44)$ & & $1.09(0.28)$ & \\
\hline \multicolumn{11}{|l|}{ Dinner } \\
\hline No & 7 & $25.20 \pm 15.11$ & 14 & $63.37 \pm 20.07$ & $-6.24(0.000)$ & 14 & $25.00 \pm 25.65$ & 27 & $66.06 \pm 20.81$ & $-5.53(0.000)$ \\
\hline Yes & 111 & $27.86 \pm 13.88$ & 280 & $71.37 \pm 15.87$ & $-18.14(0.000)$ & 101 & $29.60 \pm 17.49$ & 254 & $69.79 \pm 19.52$ & $-18.01(0.000)$ \\
\hline$t(\mathrm{p})$ & & $-0.45(0.65)$ & & $-1.58(0.12)$ & & & $-0.87(0.39)$ & & $-0.94(0.35)$ & \\
\hline \multicolumn{11}{|l|}{ Mid-Day Snack } \\
\hline No & 17 & $24.65 \pm 14.90$ & 43 & $62.10 \pm 19.93$ & $-6.08(0.000)$ & 17 & $28.09 \pm 19.03$ & 18 & $59.69 \pm 15.88$ & $-5.35(0.000)$ \\
\hline Yes & 101 & $29.56 \pm 17.97$ & 252 & $64.10 \pm 19.89$ & $-17.99(0.000)$ & 98 & $29.21 \pm 18.61$ & 263 & $70.10 \pm 19.72$ & $-17.79(0.000)$ \\
\hline$t(\mathrm{p})$ & & $-1.25(0.21)$ & & $-0.61(0.54)$ & & & $-0.23(0.82)$ & & $-2.19(0.03)$ & \\
\hline
\end{tabular}

Table 8. Continued.

\begin{tabular}{|c|c|c|c|c|c|}
\hline & \multicolumn{5}{|c|}{ Combined } \\
\hline & \multicolumn{2}{|c|}{ Not Having Meal } & \multicolumn{2}{|c|}{ Having Meal } & \multirow{2}{*}{$\begin{array}{l}t \\
\mathbf{p}\end{array}$} \\
\hline & n & Mean \pm SD & n & Mean \pm SD & \\
\hline \multicolumn{6}{|l|}{ Breakfast } \\
\hline Yes & 145 & $27.73 \pm 18.22$ & 412 & $66.90 \pm 20.32$ & $-21.65(0.000)$ \\
\hline$t(\mathrm{p})$ & & $-0.39(0.70)$ & & $-0.26(0.79)$ & \\
\hline Yes & 139 & $27.75 \pm 16.24$ & 409 & $66.94 \pm 20.49$ & $-20.81(0.000)$ \\
\hline$t(\mathrm{p})$ & & $-0.63(0.53)$ & & $-0.30(0.77)$ & \\
\hline \multicolumn{6}{|c|}{ Number of Meals per day } \\
\hline Less than 3 meals/day & 28 & $27.06 \pm 16.87$ & 76 & $66.51 \pm 20.31$ & $-10.02(0.000)$ \\
\hline 3 or more meals/day & 205 & $28.04 \pm 17.64$ & 500 & $66.83 \pm 17.46$ & $-24.55(0.000)$ \\
\hline$t(\mathrm{p})$ & & $-0.29(0.78)$ & & $-0.13(0.90)$ & \\
\hline$t(\mathrm{p})$ & & $-2.36(0.02)$ & & $-3.03(0.003)$ & \\
\hline \multicolumn{6}{|l|}{ Dinner } \\
\hline No & 21 & $25.95 \pm 22.07$ & 41 & $66.25 \pm 20.02$ & $-7.75(0.000)$ \\
\hline Yes & 212 & $27.30 \pm 16.40$ & 534 & $68.07 \pm 19.28$ & $-25.28(0.000)$ \\
\hline$t(\mathrm{p})$ & & $-0.35(0.73)$ & & $-0.51(0.61)$ & \\
\hline \multicolumn{6}{|l|}{ Mid-Day Snack } \\
\hline No & 34 & $26.89 \pm 16.97$ & 61 & $61.39 \pm 18.73$ & $-8.42(0.000)$ \\
\hline Yes & 199 & $28.82 \pm 16.84$ & 515 & $67.17 \pm 20.01$ & $-25.11(0.000)$ \\
\hline$t(\mathrm{p})$ & & $0.61(0.54)$ & & $-2.15(0.03)$ & \\
\hline
\end{tabular}

When considering the social factors, we found that they had a significant effect on math grades in the combined sample and in boy and girls separately, except for child's order of birth (Table 9). 
Table 9. Math Test School Achievement Scores for Boys, Girls and the Combined Group vs. School Meal and some Social Factors.

\begin{tabular}{|c|c|c|c|c|c|c|c|c|c|c|}
\hline & \multicolumn{5}{|c|}{ Boys } & \multicolumn{5}{|c|}{ Girls } \\
\hline & \multicolumn{2}{|c|}{ Not Having Meal } & \multicolumn{2}{|c|}{ Having Meal } & \multirow{2}{*}{$\begin{array}{l}t \\
\mathbf{p}\end{array}$} & \multicolumn{2}{|c|}{ Not Having Meal } & \multicolumn{2}{|c|}{ Having Meal } & \multirow{2}{*}{$\begin{array}{l}t \\
\mathrm{p}\end{array}$} \\
\hline & $\mathrm{n}$ & Mean \pm SD & n & Mean \pm SD & & $\mathbf{n}$ & Mean \pm SD & n & Mean \pm SD & \\
\hline \multicolumn{11}{|c|}{ Child Order of Birth } \\
\hline Child $3^{\text {rd }}$ or more & 49 & $26.17 \pm 16.02$ & 159 & $60.63 \pm 21.09$ & $-10.53(0.000)$ & 51 & $31.91 \pm 18.77$ & 151 & $68.55 \pm 19.22$ & $-11.84(0.000)$ \\
\hline Child $1^{\text {st }}-2^{\text {nd }}$ & 69 & $24.78 \pm 14.32$ & 136 & $67.54 \pm 17.72$ & $-17.36(0.000)$ & 64 & $26.76 \pm 18.27$ & 130 & $70.46 \pm 20.15$ & $-14.64(0.000)$ \\
\hline$t(\mathrm{p})$ & & $0.49(0.62)$ & & $-3.02(0.003)$ & & & $1.49(0.14)$ & & $-0.81(0.42)$ & \\
\hline \multicolumn{11}{|l|}{ Family Size } \\
\hline Large & 56 & $26.70 \pm 15.46$ & 233 & $62.53 \pm 20.42$ & $-12.31(0.000)$ & 53 & $29.95 \pm 18.27$ & 229 & $69.20 \pm 19.78$ & $-13.20(0.000)$ \\
\hline Small & 62 & $24.15 \pm 14.59$ & 62 & $68.61 \pm 16.97$ & $-15.64(0.000)$ & 62 & $28.27 \pm 18.98$ & 52 & $70.47 \pm 19.15$ & $-11.78(0.000)$ \\
\hline$t(\mathrm{p})$ & & $0.92(0.36)$ & & $-2.15(0.03)$ & & & $0.48(0.63)$ & & $-0.42(0.68)$ & \\
\hline \multicolumn{11}{|c|}{ Mother Education (Low-High) } \\
\hline Low & 47 & $27.92 \pm 13.12$ & 240 & $62.44 \pm 19.10$ & $-11.85(0.000)$ & 52 & $33.17 \pm 17.66$ & 229 & $68.51 \pm 18.51$ & $-12.53(0.000)$ \\
\hline High & 71 & $23.66 \pm 15.98$ & 55 & $69.80 \pm 22.19$ & $-13.56(0.000)$ & 63 & $25.63 \pm 18.79$ & 52 & $73.52 \pm 23.79$ & $-12.06(0.000)$ \\
\hline$t(\mathrm{p})$ & & $1.52(0.13)$ & & $-2.50(0.013)$ & & & $2.20(0.03)$ & & $-1.67(0.09)$ & \\
\hline \multicolumn{11}{|c|}{ Father Education (Low-High) } \\
\hline Low & 35 & $29.43 \pm 15.28$ & 220 & $61.24 \pm 19.07$ & $-9.39(0.000)$ & 38 & $31.32 \pm 19.26$ & 192 & $68.23 \pm 18.29$ & $-11.27(0.000)$ \\
\hline High & 83 & $23.64 \pm 14.63$ & 75 & $71.36 \pm 20.38$ & $-17.02(0.000)$ & 77 & $27.92 \pm 18.28$ & 89 & $72.03 \pm 22.16$ & $-13.86(0.000)$ \\
\hline$t(\mathrm{p})$ & & $1.94(0.06)$ & & $-3.90(0.000)$ & & & $0.92(0.36)$ & & $-1.51(0.13)$ & \\
\hline \multicolumn{11}{|c|}{ Father Job (Low-High) } \\
\hline Low & 77 & $27.92 \pm 18.28$ & 89 & $72.03 \pm 22.16$ & $-13.86(0.000)$ & 51 & $32.30 \pm 20.13$ & 196 & $66.82 \pm 19.31$ & $-11.27(0.000)$ \\
\hline High & 71 & $23.59 \pm 14.99$ & 100 & $67.64 \pm 20.79$ & $-15.25(0.000)$ & 64 & $26.45 \pm 16.98$ & 85 & $75.48 \pm 19.17$ & $-16.22(0.000)$ \\
\hline$t(\mathrm{p})$ & & $1.59(0.12)$ & & $-2.39(0.02)$ & & & $1.69(0.09)$ & & $-3.46(0.001)$ & \\
\hline \multicolumn{11}{|c|}{ Mother Job (Low-High) } \\
\hline Low & 82 & $25.33 \pm 14.32$ & 276 & $63.21 \pm 19.80$ & $-16.11(0.000)$ & 78 & $31.15 \pm 18.74$ & 263 & $69.46 \pm 18.81$ & $-15.81(0.000)$ \\
\hline High & 36 & $25.42 \pm 16.65$ & 19 & $72.61 \pm 19.42$ & $-9.43(0.000)$ & 37 & $24.59 \pm 17.72$ & 18 & $69.06 \pm 29.99$ & $-6.91(0.000)$ \\
\hline$t(\mathrm{p})$ & & $-0.03(0.98)$ & & $-2.00(0.05)$ & & & $1.78(0.08)$ & & $0.09(0.93)$ & \\
\hline
\end{tabular}

Table 9. Continued.

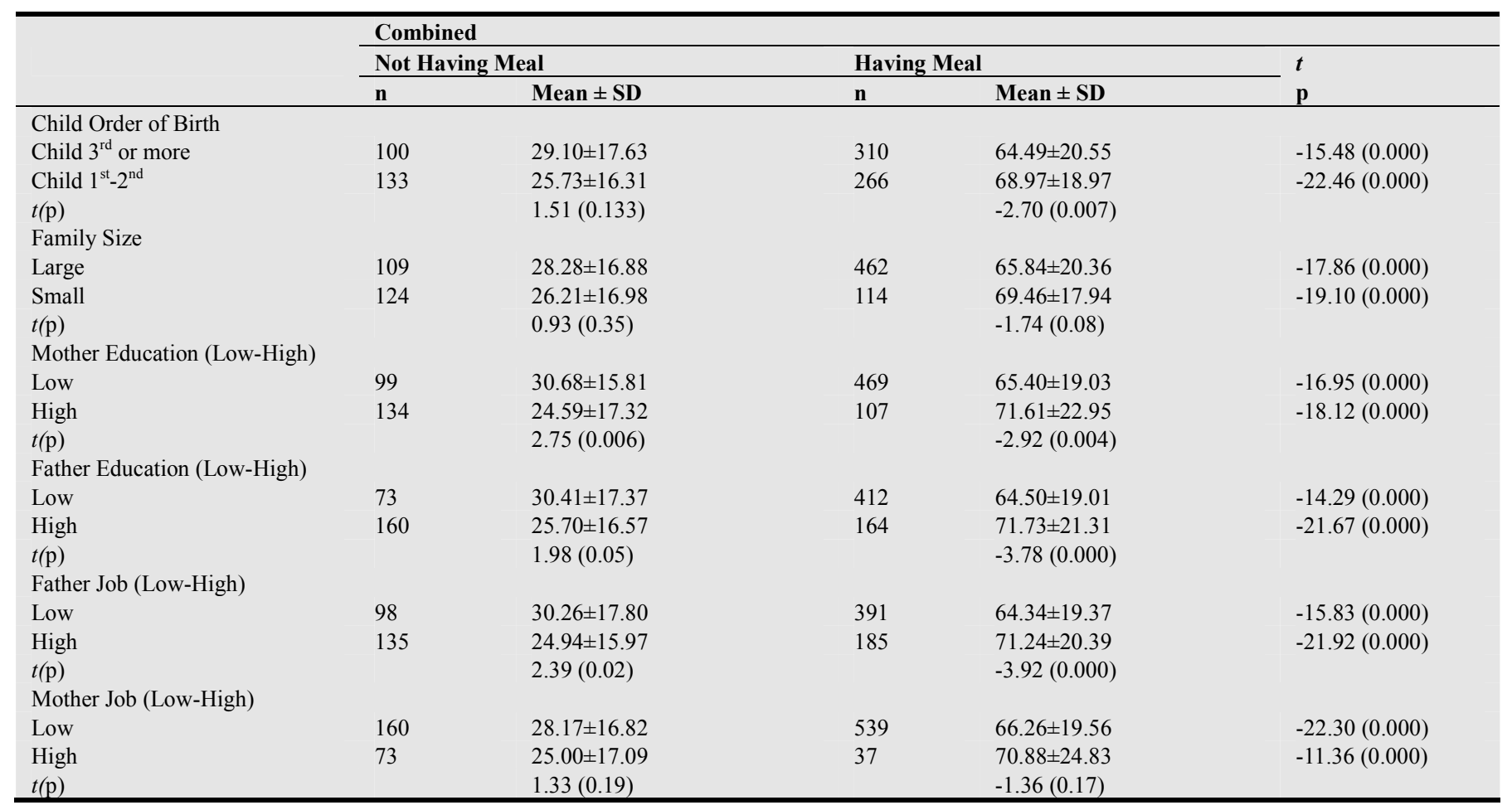

Our findings indicated unsatisfactory academic achievement in terms of test scores, which did not significantly differ between boys and girls who did not have a school meal. But when considering the effect of the school 
meal, we noticed a significant difference between boys and girls; girls had better grades in both math and total score. Our results agree with work by Tan, Lane, and Lassibille (1999) who evaluated the impact of the school feeding program in the Philippines. The authors found that school feeding, either alone or with parent-teacher partnerships, had a positive and statistically significant effect on mathematics test scores. Also, Vermeersch and Kremer (2004) found that the school meals increased test scores in schools where the teacher was experienced.

Step-wise multiple linear regression analysis in table (10) shows that school meal, father's job, sex, regularity of meals and a mid-day snack were found to be significantly $(p<0.001)$ associated with school achievement. While, math grades were associated with school meal, sex, regularity of meals and father's job.

Table 10. Order of Variables Associated with School Achievement and Math Grades According to Step-wise Regression.

\begin{tabular}{lllll}
\hline Dependent variable & Order of predictor variables ${ }^{\mathbf{b}}$ & $\mathbf{R}^{\mathbf{2}} \mathbf{\%}$ & F & P value \\
\hline School achievement & School meal, father job, sex, regularity of meals and mid day snack & 27.0 & 58.42 & $<0.001$ \\
Math grades & School meal, sex, regularity of meals and father job & 53.0 & 227.68 & $<0.001$ \\
\hline
\end{tabular}

Multiple regression analysis ${ }^{\text {a }}$

a: All studied variables ( social, dietary behaviour and indicators of nutritional status) entered in the analysis as independent variables.

b: Variables were presented in descending order according to the value of the regression coefficients.

\section{Discussion}

Taras (2005) who reviews research from published studies on the association between nutrition among school-aged children and their performance in school and on tests of cognitive functioning, finds the following: Children with iron deficiencies sufficient to cause anemia are at a disadvantage academically, unless they receive iron therapy. Students with mild iron deficiencies and no anemia do not routinely exhibit problems in cognition or academic performance. Food insufficiency is a serious problem affecting children's ability to learn.

Research indicates that school meal programs contribute to improving academic performance and to providing healthier dietary behavior in participating students (Gross and Cinelli, 2004). Kim et al., 2003 found that the regularity of breakfast and lunch was associated with academic performance. Also, a weak association of having a regular dinner with academic performance was seen by them. The finding of our study demonstrates that consumption of school meal has a positive impact on mathematics achievements; other nutritional factors are consumption of 3 or more meals per day and eating breakfast. This finding was demonstrated by other studies done in different countries (Grantham-McGregor, 2005; McCann \& Ames, 2007).

It has been previously reported that short-term breakfast omission diminishes short-term memory in children (Simeon, 1998, Pollitt et al., 1998, Benton \& Patker, 1998, Adrogué and Orlicki, 2013).In the long-term, school breakfast consumption was shown to improve school attendance and scholastic achievement of children (Meyers et al., 1989, Powell et al., 1983). Also, eating meals at a regular time may be a reflection of food security (Wolfe \& Frongillo, 2001). In addition, meal regularity also may reflect family care, (Kleinman et al., 1998; Murphy et al., 1998) investigators found that school-age children identified as hungry and/or at risk of hunger were more likely to have impaired psychosocial function and academic outcome. Using data from NHANES III, Alaimo and coworkers, 2001 also found that family food insufficiency was associated with school-age children's academic and psychosocial development. Alaimo et al., 2001 outlined several possible mechanisms for their results that could also be relevant to explaining the relation between meal irregularity and poorer school performance: 1) poorer health status, 2) irritability, distractibility, and emotional changes, 3) anxiety resulting from feelings of deprivation, 4) parental stress, anxiety, and behavior, and 5) family functioning.

Giving children a daily breakfast or a meal at school improves their scholastic achievement through several mechanisms, for example by activation of the brain, which is sensitive to short-term variations in the availability of nutrient supplies (Grantham-McGregoet al., 1998; Pollit, 1995).

This indication is particularly strong for undernourished children. Meals at school also play important roles in alleviating short-term hunger in malnourished or even wellnourished children, motivate parents (especially among the poor) to enroll their children in school and have them attend regularly, address micronutrient deficiencies among children and increase community participation and involvement in schools (Del Rosso, 1999). Achamet al., 2012 finding on the relationship between supplying breakfast and midday meals with academic achievement should be given credibility as it has provided a picture of the experience of rural schoolchildren in Uganda. Also, regular attendance is closely related to scholastic achievement. They found that an attendance rate above $70 \%$ was significantly positively associated with achievement, corroborating findings by others (Del Rosso\&Marek1996).

Evidence of the importance of parental education in shaping children's school performance is mixed. In Egypt, student performance was marginally, but positively, influenced by the father's educational level but the mother's education did not play a significant role (Hanushek \& Lavy, 1994).

It is not surprising that the SFP improves both nutritional status and test scores. Although the SF biscuits look like typical biscuits, they are not. These high-energy biscuits provide 75 percent of the recommended daily allowance of vitamins and minerals for a primary school-age child. Most of the program 
children had been eating these biscuits every school day for more than a year before the survey (Ahmed, 2004).

Ahmed (2004) evaluated the impact of the in-school meal program in Bangladesh on test scores using data on achievement test scores for 1,648 students in grade5 attending primary school. Using an econometric specification to isolate the effects of the program, he found that students in program schools score 15.7 percent higher than did students in the control schools. This increase is statistically significant. He further decomposed this increase into the three subjects that make up the total score and found that the improvement was due mainly to an increase in the mathematics test score.

Student's gender strongly affects their academic performance, with girls performing better in the subjects of Mathematics, and English as well as cumulatively. Kamogawa, 2003 and Hamid Jan et al., 2011 reported that female Malaysian students earned better grades than males. Girls usually show more efforts leading towards better grades at school (Ceballo, McLoyd \&Toyokawa, 2004).

\section{Conclusion}

School snack in this study has the opportunity to reduce the short-term hunger in the classroom so that the students can concentrate and learn better. Providing students with important micronutrients especially iron, on a regular basis through daily intake of school snacks, results in improvement in nutrient intake status school performance. School breakfast should be considered for both low-income children and children receiving little attention at home. Meanwhile, dietary behavior especially having breakfast beside meals is considered as enabling environment' supportive to having school meal. Accordingly, the ongoing process of the National School Feeding Program, should reach a sustainable mechanism of providing fortified school meals to all children thus helping extend the school feeding program to include the increasing number of families and their children now under threat of falling below the poverty line, who have been exposed to food hardship as a result of the recent high food prices.

School meal should be one of multiple interventions designed to improve the health and development of poor and disadvantaged children. It should be combined with other interventions as Awareness nutrition and health education programs are recommended to educate caregivers how to increase caloric and essential micro nutrients (food for the brain) of their children diets, using available food in their communities.

\section{References}

[1] Acham, H., Kikafunda, J. K.; Malde, M. K.; OldewageTheron, W. H. \& Egal, Abdul Kadir A. (2012). Breakfast, midday meals and academic achievement in rural primary schools in Uganda: implications for education and school health policy. Food \& Nutrition Research, 56, 11217 -11229.
[2] Adrogué, C and Orlicki, M. E (2013) Do In-School Feeding Programs Have Impact on Academic Performance and Dropouts? The Case of Public Argentine Schools. Education Policy Analysis Archives, 21(50), 1-23.

[3] Ahmed, AU. (2004). Impact of feeding children in school: evidence from Bangladesh. Washington, DC: International Food Policy Research Institute.

[4] Alaimo, K; Olson, CM \& Frongillo, EA. (2001).Food insufficiency and American school-aged children's cognitive, academic, and psychosocial development. Pediatr; 108: 44-53.

[5] Ani, C. \& Grantham-McGregor, S. (1999). The effects of breakfast onchildren's educational performance, attendance and class room behaviour. In: Fit for School (Donovan N, Street C, eds), 11-17. New Policy Institute, London.

[6] Benton, D. \& Patker, PY. (1998). Breakfast, blood glucose, and cognition. Am J. Clin. Nutr; 67: 772S-778S.

[7] Bhaskaram, P. (2001). Immunobiology of mild micronutrient deficiencies. British Journal of Nutrition, 85, S75-S80.

[8] Ceballo, R., McLoyd, V., \&Toyokawa, T. (2004). The influence of neighborhood quality on adolescents' educational values and school efforts. Journal of Adolescent Research, 19(6), 716-739.

[9] Del Rosso, JM \& Marek, T. (1996). Class action: improving school performance in the developing world through better health and nutrition. Washington, DC: World Bank; (1996).

[10] Del Rosso JM. (1999). School feeding programs: improving effectiveness and increasing the benefit to education. A guide for Program managers. The Partnership for Child Development, 1999.

[11] Ekaju, J. (2011). An investigation into the relationship between the 1997 Universal Primary Education (UPE) Policy and regional poverty and educational inequalities in Uganda (19972007). Thesis, University of Glasgow, 2011.

[12] Gorman, KS (1995). Malnutrition and cognitive development: evidence from experimental/quasi-experimental studies among the mild-to moderately malnourished. Journal of Nutrition, $125,2239 \mathrm{~S}-44 \mathrm{~S}$.

[13] Grantham-McGregor S.(2005). Can the provision of breakfast benefit school performance? Food Nutr Bull. Jun; 26 (2 Suppl 2): S144-58.

[14] Grantham-McGregor, SM; Chang, S. \& Walker, SP. (1998) Evaluation of school feeding programmes: some Jamaican examples. Am J Clin. Nutr; 67: 785S-9S.

[15] Grantham-McGregor, S. (2002). Linear growth retardation and cognition. Lancet, 359- 542.

[16] Grantham-McGregor, S.(1995). A review of studies of the effect of severe malnutrition on mental development. Journal of Nutrition, 125, 2233S-8S.

[17] Grantham-McGregor, S.; Walker, S. \& Chang, S. (2000). Nutritional deficiencies and later behavioural development. Proceedings of the Nutrition Society, 59, 47-54.

[18] Gross, SM and Cinelli, B (2004). Coordinated school health program and dietetics professionals: partners in promoting healthful eating. Journal of the American Dietetic Association, 10 (5), 793-798. 
[19] Hamid Jan JMt, Amal K Mitra'z, Hasmiza H', Pim CD, Ng LOr \& Wan Manan WM' (2011). Effect of Gender and Nutritional Status on Academic Achievement and Cognitive Function among Primary School Children in a Rural District in Malaysia. Mal J Nutr, 17(2), 189-200.

[20] Hanushek, EA \& Lavy, V. (1994). School quality, achievement bias, and dropout behaviour in Egypt, LSMS Working Paper, No. 107. Washington, DC: World Bank.

[21] Kamogawa, A, (2003). Higher education reform: Challenges towards a knowledge society in Malaysia, $<$ http://unpan1.un.org/intradoc/groups/public/documents/ APCITY/UNPAN025531.pdf [Accessed 28 May 2014].

[22] Kleinman, RE, Murphy, JM, Little, M, Pagano, M, Wehler, CA, Regal, K, Jellinek, MS.(1998). Hunger in children in the United States: potential behavioral and emotional correlates. Pediatr; 101:e3.

[23] Levitsky, D. \& Strupp, B. (1995). Malnutrition and the brain: changing concepts, changing concerns. Journal of Nutrition, $125,2212 \mathrm{~S}-20 \mathrm{~S}$.

[24] McCann Joyce, C and Ames Bruce, N. (2007). An overview of evidence for a causal relation between iron deficiency during development and deficits in cognitive or behavioral function. Am J ClinNutr; 85, 931-45.

[25] Meyers, AF; Sampson, AE; Weitzman, M; Rogers, BL \& Kayne, H. (1989). School breakfast program and school performance. Am J Dis Child; 143: 1234-1239.

[26] Murphy, J; Wehler, C; Pagano, M; Little, M; Kleinman, R \& Jellinek M. (1998). Relationship between hunger and psychosocial functioning in low-income American children. $J$ Am Acad Child Adolesc Psychiatry; 37: 163-170.

[27] Murphy, J. M.; Pagano, M. E.; Nachmani, J.; Sperling; P.; Kane, S. \& Kleinman, R. E. (1998). The Relationship of School Breakfast to Psychosocial and Academic Functioning. ARCH PEDIATR ADOLESC MED, 152, 899-907.

[28] Pollit, E. (1995). Does breakfast make a difference in school? $J$ Am Diet Assoc; 95(10): 11349.

[29] Pollitt, E.; Cueto, S \& Jacoby, ER. (1998). Fasting and cognition in well and undernourished schoolchildren: a review of three experimental studies. Am J Clin. Nutr; 67: 779S-784S.
[30] Pollitt, E; Golub, M; Gorman, K; Grantham-McGregor, S; Levitsky, D; Schurch, B; Strupp, B \& Wachs, T. (1996). A reconceptualization of the effects of undernutrition on children's biological, psychosocial, and behavioral development. Social Policy Rep; 10: 1-21.

[31] Powell, C; Grantham-McGregor, SM \& Elston, M. (1983).An evaluation of giving the Jamaican government school meal to a class of children. Hum. Nutr. Clin. Nutr; 37C: 381-388.

[32] Powell, CA. \& Grantham-McGregor, SM. (1980). The association between nutritional status, school achievement and school attendance in twelve-year-old children at a Jamaican school. W Indian Med J.; 29: 247-53.

[33] Sewnet, M. (1995). Some factors affecting scholastic achievement ofelementary school pupils. Thesis, School of Graduate Studies,Addis Ababa University.

[34] Simeon, DT. (1998). School feeding in Jamaica: a review of its evaluation. Am J Clin. Nutr; 67: 790S-794S.

[35] Tan, J.-P.; Lane, J. and Lassibille, G. (1999). Student outcomes in Philippine elementary schools: An evaluation of four experiments. World Bank Economic Review, 13 (3): 493-508.

[36] Taras, H. (2005). Nutrition and Student Performance at School. Journal of School Health, 75(6), 199-213

[37] Trochim, W. M. Research Methods Knowledge Base. Accessed online: http://trochim.human.cornell.edu/kb/index.htm.

[38] Wachs, T.(1995). Relation to mild-to-moderate malnutrition to human development: correlational studies. Journal of Nutrition, 125, 2245-54.

[39] Wolfe, WS \& Frongillo, EA. (2001). Building household foodsecurity measurement tools from the ground up. Food Nutr Bull; 22:5-12.

[40] World Bank. Learning for all: investing in people's knowledge and skills to promote development: World Bank Group Education Strategy 2020 Executive Summary, April 2011.http://siteresources.worldbank.org/EDUCATION/Resour ces/ESSU/Education_Strategy 2020 Executive Summary 4 12 2011.pdf [cited 8 October 2011]. 